\title{
Various Covering Spectra for Complete Metric Spaces
}

\author{
Christina Sormani * Guofang Wei ${ }^{\dagger}$
}

\begin{abstract}
Here we study various covering spectra for complete noncompact length spaces with universal covers (including Riemannian manifolds and the pointed Gromov Hausdorff limits of Riemannian manifolds with lower bounds on their Ricci curvature). We relate the covering spectrum to the (marked) shift spectrum of such a space. We define the slipping group generated by elements of the fundamental group whose translative lengths are 0 . We introduce a rescaled length, the rescaled covering spectrum and the rescaled slipping group. Applying these notions we prove that certain complete noncompact Riemannian manifolds with nonnegative or positive Ricci curvature have finite fundamental groups. Throughout we suggest further problems both for those interested in Riemannian geometry and those interested in metric space theory.
\end{abstract}

\section{Introduction}

The covering spectrum of a compact Riemannian manifold or length space captures the metric properties needed to obtain topological information about the given space. In prior work of the authors [36, we applied the covering spectrum to determine the properties of the fundamental group of a compact metric space and to determine whether that space has a universal cover. We proved the covering spectrum is determined by the marked length spectrum and the elements of the covering spectrum lie in the half length spectrum. Then de Smit, Gornet and Sutton developed a means of producing pairs of compact manifolds which have the same covering spectrum in 12. They produced pairs of compact manifolds with the same Laplace spectrum that have different covering spectra in 12 [13. This is intriguing in light of the work of Colin de Verdiere and DuistermaatGuillemin relating the length and Laplace spectra of compact Riemannian manifolds [10, 15]. A recent extension of the notion of covering spectrum to a larger class of spaces which is called the critical spectrum has been studied by Wilkins, Plaut, Conant, Curnutte, Jones, Pueschel and Walpole [4] [29] [2] [11. The key definitions, theorems and examples are reviewed in Section 2.

In this paper we are concerned with the covering spectra and other related spectra on complete noncompact Riemannian manifolds and length spaces. Prior work in this direction has been conducted by the authors in [37] where we developed the notion of the cut off covering spectrum. The cut off covering spectrum effectively removed information about the space outside of large balls thus enabling us to extend a number of our prior results. However data about the behavior of the space at infinity was lost in the process.

Here we develop new spectra designed to capture the properties of complete noncompact Riemannian manifolds at infinity. More generally, we assume all of our metric spaces, $X$, are complete noncompact length spaces as in Definition 2.1 and that they have universal covers, $\tilde{X}$, in the sense that the universal cover is a cover of all covering spaces (c.f. [38). We do not assume the universal covering is simply connected. Recall that the Gromov-Hausdorff limits of complete noncompact Riemannian manifolds with uniform lower bounds on their Ricci curvature were proven to have universal covering spaces in [35. So all results in this paper apply to these essential limit spaces that

\footnotetext{
*Partially supported by NSF Grant \# DMS-1006059 and a PSC CUNY Research Award

${ }^{\dagger}$ Partially supported by NSF Grant \# DMS-1105536.
} 
have been explored extensively by Cheeger, Colding, Ding, Ennis, Honda, Menguy, Munn, Naber, Ohta, Tian, the authors and many others (c.f. [5 $9,14,16,18,22,20]$ ).

In the final section of the paper, we apply our spectra to prove special cases of Milnor's conjecture that the fundamental group of a complete noncompact manifold with nonnegative Ricci curvature is finitely generated 21]. Prior work in this direction has been conducted by Li, Anderson, Wilking and the first author 1] 19 31 40. In fact we prove complete noncompact manifolds with certain spectral properties that have positive or nonnegative Ricci curvature have a finite fundamental group [Theorems 1.1 and 1.2 respectively]. So one might prefer to view these as extensions of Myers' Theorem [23] that compact manifolds with positive Ricci curvature have finite fundamental groups. Before we specialize to Riemannian manifolds, we first need to extend our theorems about the covering spectrum proven in [36] to complete noncompact length spaces and introduce our new covering spectra.

In Section 3, we extend our results in [36] relating the covering spectrum and the length spectrum of a compact length space to the complete noncompact setting. We introduce the (marked) shift spectrum [Definition 3.1] which captures the lengths of elements of the fundamental group in the complete noncompact setting and agrees with the classical (marked) length spectrum on compact length spaces. We prove Theorem 3.2 that the the marked shift spectrum determines the covering spectrum on such spaces (extending Theorem 4.7 of [36]). We prove the covering spectrum is a subset of the closure of the half shift spectrum in Theorem 3.3. In the compact setting this was proven in [36] without requiring a closure, but in the complete noncompact setting we show this is necessary with Example 3.1. We suggest notion of a (marked) shift spectrum that might be studied on spaces without universal covers [Remark 3.5. We close this section by discussing elements of the fundamental group whose lengths are achieved and proving Theorem 3.6 that if infinitely many such elements have their lengths achieved in a common compact set then the universal cover contains a line.

In Section 4, we extend our results in [36] relating the covering spectrum to the universal cover of a compact length space to the complete noncompact setting. We introduce the universal slipping group, $\pi_{\text {slip }}(X)$, and the universal delta cover, $\tilde{X}^{0}$ [Definitions 4.3 and 4.5 . On a compact metric space, the universal slipping group is trivial and the universal delta cover is the universal cover. In fact the authors proved that if the covering spectrum of a compact length space has a positive infimum, then the universal cover exists and is a $\delta$ cover [36. Here we present complete noncompact manifolds whose $\delta$ covers are all trivial and whose universal cover is nontrivial [Example 4.1. Every element of the fundamental group in this example is represented by a sequence of loops which slip out to infinity as their lengths decrease to 0 . We prove the universal delta cover, $\tilde{X}^{0}$, is a pointed Gromov-Hausdorff limit of $\delta$ covers as $\delta \rightarrow 0$ [Theorem [4.6. It need not be a delta cover itself [Remark 4.8], nor is it the universal cover [Remark 4.9]. However, if the covering spectrum has a positive infimum, then $\tilde{X}^{0}$ is a delta cover [Proposition 4.7. In general we show $\tilde{X}^{0}=\tilde{X} / \pi_{\text {slip }}(X)$ [Theorem 4.10]. Thus if the slipping group is empty and the covering spectrum has a positive infimum, the universal cover is a $\delta$ cover [Corollary 4.12. In Remark 4.13 we suggest that an adaption of this work to complete length spaces which are not known to have universal covers might be applied to prove the existence of a universal cover for such a space.

In Section 5, we introduce two new scale invariant covering spectrum: the basepoint dependent rescaled covering spectrum, $\operatorname{Cov} \operatorname{Spec}_{r s}^{x_{0}}(X)$, and the infinite rescaled covering spectrum, $\operatorname{CovSpec}_{r s}^{\infty}(X)$ [Definitions 5.12. Both these spectra are defined only on complete noncompact spaces using $\delta$ rescaled covering groups [Definition 5.9] whose elements have rescaled lengths [Definitions 5.1]and 5.3. defined by measuring the lengths sequences of representative loops based at points diverging to infinity. These rescaled covering spectra take values lying in $[0,1]$. We also define the rescaled slipping group, $\pi_{r s}^{\infty}(X, 0)$, which is generated by elements of the fundamental group that have representative loops diverging to infinity whose rescaled lengths converge to 0 . In the final subsection we suggest a few means of defining these spectra on spaces without universal covers. Nevertheless, we believe 
the rescaled covering spectra will prove most useful in the study of submanifolds of Euclidean space and Riemannian manifolds, both of which always have universal covers.

In Section 6, we study the asymptotic behavior of complete noncompact metric spaces using the rescaled covering spectra and the rescaled slipping group. We prove that if the rescaled length of an element of the fundamental group is strictly less than 2 , then it is represented by a sequence of loops diverging to infinity [Lemma 6.2. We prove that if $\operatorname{CovSpec}_{r s}^{\infty}(X) \subset[0,1)$ then the cut off covering spectrum of [37] is trivial [Theorem [6.3. These two spectra are recording very different metric topological information about a space. Next we compute the rescaled covering spectra of one sheeted hyperboloids [Example 6.1] and doubly warped products [Theorem 6.6]. We close with a conjecture about the rescaled covering spectra of manifolds with linear diameter growth [Conjecture 6.8.

The notions and theorems in Sections 2-6 are then applied in the final section to complete noncompact Riemannian manifolds with curvature bounds. First we study manifolds with nonnegative sectional curvature, whose topology can be understood because such manifolds have a compact soul. We prove a noncompact versions of Myers' Theorem:

Theorem 1.1 If $M$ has positive Ricci curvature and $K \subset M$ is compact, then only finitely many elements of the fundamental group have their lengths achieved within $K$.

This theorem is proven by constructing a line in the universal cover and applying the CheegerGromoll Splitting Theorem [7. Note that in the classic example of Nabonnand [24], which has positive Ricci curvature, there are infinitely many elements in the fundamental group as well, but their lengths are not achieved anywhere. In a cylinder, which has nonnegative Ricci curvature, infinitely many elements can have their length achieved in a common compact set however the rescaled covering spectrum of the cylinder is trivial and the fundamental group lies in the rescaled slipping group. In fact we prove:

Theorem 1.2 If $M^{n}$ is a complete noncompact Riemannian manifold whose rescaled slipping group, $\pi_{r s}^{\infty}(M, 0)$, is trivial and whose rescaled covering spectrum, $\operatorname{CovSpec}_{r s}^{\infty}(M)$, has a positive infimum, then it has a finite fundamental group.

This theorem is proven by applying the Bishop-Gromov Volume Comparison Theorem in a style similar to Milnor's proof that finitely generated subgroups of the fundamental group have polynomial growth 21]. Note that in the example of Nabonnand the entire fundamental group lies in the rescaled slipping group. We observe that a similar statement holds when $M$ is the pointed Gromov-Hausdorff limit of manifolds with nonnegative Ricci curvature [Remark 7.14] including the tangent cone at infinity for such a space.

It is possible that the techniques applied to prove these theorems may lead to a proof of the Milnor Conjecture that the fundamental group of a manifold with nonnegative Ricci curvature is finitely generated. Our proofs apply the Cheeger-Gromoll Splitting Theorem and a Milnor style application of the Bishop Gromov Volume Comparison Theorem. Other techniques for controlling the fundamental groups of such manifolds that appear in work of Anderson, Li, Wilking and the first author in [1] 19] 31, 40, have not yet been applied in combination with our new ideas. Further suggested problems are stated in the final remarks of the paper.

The authors would like to thank Ruth Gornet, Carolyn Gordon and Shing-Tung Yau for their interest in the covering spectrum and Burkhard Wilking for suggesting an analysis of $\mathbb{S}^{3} \times \mathbb{R}^{4} / \operatorname{Pin}(2)$.

\section{Background}

In this section we review our prior work and that of others taking advantage of the simplified definitions one can use when there is a universal cover. 
Definition 2.1 A complete length space is a complete metric space such that every pair of points in the space is joined by a length minimizing rectifiable curve. The distance between the points is the length of that curve. A compact length space is a compact complete length space. (c.f. 4]).

Note that complete Riemannian manifolds are complete length spaces by the Hopf-Rinow Theorem. As in Riemannian geometry, we define geodesics as follows (c.f. [4] [33]):

Definition 2.2 A geodesic $\gamma: I \rightarrow X$, is a locally length minimizing curve:

$$
\forall t \in I \quad \exists \varepsilon_{t}>0 \text { such that } d_{X}(\gamma(t-\varepsilon), \gamma(t+\varepsilon))=L(C([t-\varepsilon, t+\varepsilon])) .
$$

The length spectrum of a Riemannian manifold in a classical notion studied by many people over the years (c.f. [10]). Here we recall the definition on a complete length space (c.f. [33]):

Definition 2.3 The length spectrum is defined:

$$
\operatorname{Length}(X)=\left\{L(\gamma): \gamma: \mathbb{S}^{1} \rightarrow X\right\}
$$

where $\gamma$ is a closed geodesic.

Recall that on a metric space, $X$, with a universal cover, $\tilde{X}$, every element, $g$, in the fundamental group, $\pi_{1}(X)$, can be thought of as a deck transform of the univeral cover $g: X \rightarrow X$. Thus every element has a well defined length

$$
L(g)=\inf _{x \in X} d_{\tilde{X}}(g \tilde{x}, \tilde{x}) \in[0, \infty),
$$

where $\tilde{x}$ is an arbitrary lift of $x$ to the universal cover. On complete noncompact spaces the length in (2.3) might not be achieved and could be 0. It is sometimes referred to as the translation length in CAT(0) geometry (c.f. 3]). On compact manifolds this infimum is achieved at some $x_{0}$ and any minimal geodesic running between $\tilde{x}_{0} \in X$ and $g \tilde{x}_{0}$ can be seen to project to a geodesic loop, $\sigma: \mathbb{S}^{1} \rightarrow X$.

Definition 2.4 The marked length spectrum is the map:

$$
L: \pi_{1}(X) \rightarrow[0, \infty)
$$

where $L(g)$ is defined as in (2.3). The image $L\left(\pi_{1}(X)\right)$ is a subset of the length spectrum on a compact manifold because it is achieved by a closed geodesic.

Let $\pi_{1}(X, \delta)$ be the subgroup of the fundamental group generated by elements of length $<2 \delta$ :

$$
\pi_{1}(X, \delta)=\left\langle g \in \pi_{1}(X): L(g)<2 \delta\right\rangle .
$$

In 37] Thm 2.14, the authors prove that the $\delta$ cover, $\tilde{X}^{\delta}$ of a metric space with a universal cover can be found by taking

$$
\tilde{X}^{\delta}=\tilde{X} / \pi_{1}(X, \delta) .
$$

The covering spectrum of metric space (initially defined in [36]) measures the size of one dimensional holes in the space:

Definition 2.5 Given a complete length space $X$, the covering spectrum of $X$, denoted CovSpec $(X)$ is the set of all $\delta>0$ such that

$$
\tilde{X}^{\delta} \neq \tilde{X}^{\delta^{\prime}}
$$

for all $\delta^{\prime}>\delta$. 
When $X$ has a universal cover then $\delta \in \operatorname{Cov} \operatorname{Spec}(X)$ iff

$$
\pi_{1}(X, \delta) \neq \pi_{1}\left(X, \delta^{\prime}\right)
$$

for all $\delta^{\prime}>\delta$. Note that $\delta$ covers are monotone in the sense that if $\delta_{1}<\delta_{2}$ then $\tilde{X}^{\delta_{1}}$ covers $\tilde{X}^{\delta_{1}}$. In a compact manifold, where all lengths of elements are positive, any covering space is covered by a $\delta$ cover [34. In [36], the authors proved that the covering spectrum of a compact length space is a subset of the half length spectrum:

$$
\lambda \in \operatorname{CovSpec}(X) \quad \Longrightarrow \quad 2 \lambda \in \operatorname{Length}(X) .
$$

Example 2.1 Let $X$ be the $k$ torus created by taking the isometric product of $k$ circles:

$$
X=\mathbb{S}_{r_{1}}^{1} \times \mathbb{S}_{r_{2}}^{1} \times \cdots \times \mathbb{S}_{r_{k}}^{1}
$$

where $r_{1} \geq r_{2} \geq \cdots \geq r_{k}$ and $\mathbb{S}_{r}^{1}$ denotes a circle of intrinsic diameter $r$ (circumference $2 r$ ). Then if $\delta \in\left(r_{j-1}, r_{j}\right]$ we have

$$
\tilde{X}^{\delta}=\mathbb{R} \times \mathbb{R} \times \cdots \times \mathbb{R} \times \mathbb{S}_{r_{j}}^{1} \times \cdots \times \mathbb{S}_{r_{k}}^{1} .
$$

The covering spectrum is $\left\{r_{1}, r_{2}, r_{3}, \cdots, r_{k}\right\}$.

Example 2.2 If $X$ is a genus 2 surface which is a very fat figure eight with two small holes, the covering spectrum has two elements $\left\{\lambda_{1}, \lambda_{2}\right\}$ where $\lambda_{1}$ is half the length of the shortest geodesic around the smaller hole and $\lambda_{2}$ is half the length of the shortest geodesic around the other hole. These two elements then generate the entire fundamental group.

Bart de Smit, Ruth Gornet and Craig Sutton have developed a method of producing two compact manifolds with the same covering spectrum, building upon work of Sunada used to produce pairs of compact manifolds with the same Laplace spectrum in [12]. Despite the close relationship between the Laplace and Length spectrum proven by Colin de Verdiere in [10] and the close relationship between the Covering and Length spectrum proven by the authors in [35], de Smit, Gornet and Sutton have found pairs of compact Laplace isospectral manifolds with different covering spectra. They study higher dimensional manifolds in [12] and surfaces in [13.

In the complete noncompact setting, where the lengths of elements of the fundamental group are not achieved by lengths of closed geodesic loops, one still seems to have some relationship between the covering spectrum and the translative length spectrum, as indicated by the following example:

Example 2.3 Let $M^{2}$ be the warped product $\mathbb{R} \times_{f} \mathbb{S}^{1}$ where $f(x)=1+e^{-x^{2}}$. For $\delta \leq \pi$, all balls of radius $\delta$ are simply connected because $f(x) \operatorname{diam}\left(\mathbb{S}^{1}\right) \geq \pi$ for all $x$. Thus the $\delta$ cover is the universal cover, $\tilde{M}=\mathbb{R} \times_{f} \mathbb{R}$, for $\delta \leq \pi$. For $\delta>\pi$, we capture a loop $\beta$ running around $\mathbb{S}^{1}$ in a ball of radius $\delta$ where $x \circ \beta=\sqrt{-\log (\delta / \pi-1)}$. So the $\tilde{M}^{\delta}=M$ for $\delta>\pi$. Thus the covering spectrum is just $\{\pi\}$. On the other hand one can show there is no closed geodesic of length $2 \pi$ in the marked length spectrum, because any path $\gamma$ which traverses around the $\mathbb{S}^{1}$, has length

$$
L(\gamma) \geq f(\max \{x \circ \gamma\}) 2 \pi>2 \pi .
$$

Nevertheless $L(g)=2 \pi$ where $g$ is the deck transform generating the fundamental group.

In the next section we prove Theorems 3.2 and Theorem 3.3 , relating the lengths of elements of the fundamental group to the covering spectrum for all complete length spaces. 


\section{The Covering Spectrum and the Shift Spectrum}

In this section we discuss the relationship between the covering spectrum and the length spectrum. We make the following new definition which agrees with the marked length spectrum on a compact length space.

Definition 3.1 The shift spectrum, Shift $(X)$, is the collection of translative lengths $L(g)$ of elements of the fundamental group $\pi_{1}(X)$. The marked shift spectrum is the map which takes each element of the fundamental group $\pi_{1}(X)$ to its length. Two spaces $X$ and $Y$ are said to have the same marked shift spectrum if there is an isometry between their fundamental groups, $f: \pi_{1}(X) \rightarrow \pi_{1}(Y)$ such that $L(f(g))=L(g)$ for all $g \in \pi_{1}(X)$.

It has been suggested that this should be called the translative length spectrum. Since "shift" is a shorter word than "translative" and has the same meaning, we use it instead.

As seen in Example 2.3, the shift spectrum is not necessarily a subset of the length spectrum on a complete noncompact manifold. In that example, $2 \pi \in \operatorname{Shift}(M)$, but is not the length of any smoothly closed geodesic.

\subsection{The marked shift spectrum determines the covering spectrum}

We can now extend Theorem 4.7 of [36] to complete noncompact spaces.

Theorem 3.2 The marked shift spectrum of a complete length space, X, with a universal cover determines the covering spectrum of $X$.

Proof: From Definition 2.5, for $X$ with a universal cover, the covering spectrum is given by the $\delta$ where the covering groups $\pi_{1}(X, \delta)$ change, where is $\pi_{1}(X, \delta)$ is the subgroup of the group of deck transforms generated by the deck transforms of length $<2 \delta$.

So if two spaces, $X$ and $Y$, share the same shift spectrum,

$$
\pi_{1}(X, \delta)=\pi_{1}(Y, \delta)
$$

via the restricted isometry between $\pi_{1}(X)$ and $\pi_{1}(Y)$.

Suppose on the contrary that $X$ and $Y$ have the same marked shift spectrum and different covering spectra. We may assume without loss of generality that that $\delta_{0} \in \operatorname{Cov} \operatorname{Spec}(X) \backslash \operatorname{Cov} \operatorname{Spec}(Y)$. Since $\delta_{0} \notin \operatorname{Cov} \operatorname{Spec}(Y)$, then by definition, there is some $\delta_{1}>\delta_{0}$ such that

$$
\tilde{Y}^{\delta_{0}}=\tilde{Y}^{\delta_{1}} .
$$

Thus $\pi_{1}\left(Y, \delta_{1}\right)=\pi_{1}\left(Y, \delta_{0}\right)$. Applying (3.13) we get

$$
\pi_{1}\left(X, \delta_{1}\right)=\pi_{1}\left(Y, \delta_{1}\right)=\pi_{1}\left(Y, \delta_{0}\right)=\pi_{1}\left(X, \delta_{0}\right) .
$$

Thus we have

$$
\tilde{X}^{\delta_{0}}=\tilde{X}^{\delta_{1}} .
$$

and so $\delta_{0} \notin \operatorname{Cov} \operatorname{Spec}(X)$ which is a contradiction.

\subsection{The covering spectrum lies in the closure of the shift spectrum}

Recall the lower semiclosure of a set $A \subset \mathbb{R}$, denoted $C l_{\text {lower }}(A)$, is the set of all limits of nonincreasing sequences of points in $A$ (c.f. [37.). 
Theorem 3.3 The covering spectrum is a subset of the lower semiclosure of the 1/2 shift spectrum:

$$
\operatorname{CovSpec}(X) \subset C_{\text {lower }}(\{h / 2: h \subset \operatorname{Shift}(X)\}) .
$$

Before proving this, we provide an example demonstrating that the covering spectrum is not a subset of the half shift spectrum:

Example 3.1 Let $X$ be a collection of circles of intrinsic diameter $\{\pi+\pi / j: j \in \mathbb{N}\}$ joined at a common point. Let $g_{j} \in \pi_{1}(X)$ be the element represented by a loop going once around the circle of intrinsic diameter $\pi+\pi / j$. Then

$$
\pi_{1}(X, \delta)=\left\langle g_{k}, g_{k+1}, g_{k+2}, \ldots: \pi+\pi / k<\delta\right\rangle
$$

and

$$
\operatorname{CovSpec}(X)=\{\pi(1+1 / j): j \in \mathbb{N}\} \cup\{\pi\} .
$$

Here we have included $\pi$ because for $\delta=\pi, \pi_{1}(X, \delta)$ is trivial, and for $\delta^{\prime}>\pi$, there exists $k$ sufficiently large that $\pi+\pi / k<\delta^{\prime}$ and $\pi_{1}\left(X, \delta^{\prime}\right)$ is nontrivial. On the other hand, the shift spectrum is the collection of finite sums:

$$
\operatorname{Shift}(X)=\left\{\sum_{j=1}^{N} k_{j} 2 \pi(1+1 / j): k_{j} \in\{0\} \cup \mathbb{N}, N \in \mathbb{N}\right\}
$$

which contains twice every value in CovSpec $(X)$ except the value $\pi$.

Keeping this example in mind, we now prove Theorem 3.3.

Proof: Suppose on the contrary that there is a space $X$ and an element

$$
\delta_{0} \in \operatorname{Cov} \operatorname{Spec}(X) \backslash C l_{\text {lower }}((1 / 2) \operatorname{Shift}(X)) .
$$

Then there exists $\epsilon>0$ such that

$$
\left[\delta_{0}+\epsilon\right) \cap(1 / 2) \operatorname{Shift}(X)=\emptyset .
$$

By Definition 3.1,

$$
\forall g \in \pi_{1}(M), L(g) \neq\left[2 \delta_{0}, 2 \delta_{0}+2 \epsilon\right) .
$$

Thus,

$$
\pi_{1}\left(X, \delta_{0}+\epsilon / 2\right)=\pi_{1}\left(X, \delta_{0}\right),
$$

and $\delta_{0}$ is not in the covering spectrum.

Corollary 3.4 If $X$ has a closed shift spectrum, then

$$
\operatorname{CovSpec}(X) \subset \operatorname{Shift}(X) .
$$

Remark 3.5 It is possible one might achieve the same results for spaces which do not have universal covers possibly by extending the definition in (2.3) as follows:

$$
L(g)=\sup _{\tilde{X}} \inf _{C} d_{\tilde{X}}(\tilde{C}(0), \tilde{C}(1))
$$

where the supremum is taken over all regular covering spaces, $\tilde{X}$ of $X$ and the infimum is taken over all loops $C:[0,1] \rightarrow X$ freely homotopic to a representative of $g$, and where $\tilde{C}$ is a lift of $C$ to $\tilde{X}$. This leads to a natural extension of the notion of the shift spectrum. Proving the extensions would be difficult without a common cover to examine, however one might examine how we overcome this issue in the proof that the covering spectrum is contained in the half length spectrum in [36] where no universal cover is assumed. 


\subsection{Lines in universal covers}

Naturally, in a complete noncompact Riemannian manifold, some elements of the shift spectrum may lie in the length spectrum. This occurs, for example, when the lengths of elements of the fundamental group are actually achieved within a compact set. The following theorem demonstrates that this is in some sense exceptional:

Theorem 3.6 Given a complete Riemannian manifold $M$, if there are infinitely many distinct elements $g_{1}, g_{2}, \ldots, g_{i}, \ldots$ of $\pi_{1}(M)$ such that all $L\left(g_{i}\right)$ are achieved in a compact set, then the universal cover of $M$ has a line.

Proof: Let $\gamma_{i}$ be a representative of $g_{i}$ such that $L\left(\gamma_{i}\right)=L_{i}=l\left(g_{i}\right)$ and $\gamma_{i}(0) \in K$. Since all $g_{i}$ are distinct, there is a subsequence $L_{j} \rightarrow \infty$. Fix a lift of $K, \tilde{K}$ in the universal cover. Let $\tilde{\gamma}_{j}$ be a lift of $\gamma_{j}$ with $\tilde{\gamma}_{j}(0) \in \tilde{K}$. Since $L\left(\gamma_{j}\right)=l\left(g_{j}\right), g_{j}$ is a translation along $\tilde{\gamma}_{j}$, and $\tilde{\gamma}_{j}$ is minimal on any subinterval of length $L_{j}$. In particular $\tilde{\gamma}_{j}$ is minimal on $\left[-L_{j} / 2, L_{j} / 2\right]$. Since $\tilde{\gamma}_{j}(0) \in \tilde{K}$ and $\tilde{K}$ is compact, $\tilde{\gamma}_{j}$ converges to a line.

\section{The Covering Spectrum and the Universal Cover}

On a compact length space, $\delta$ covers were used to prove the existence of a universal cover. In fact the universal cover is a $\delta$ cover for a sufficiently small $\delta$ [34], and the universal cover of a compact length space exists iff its covering spectrum is finite [36]. The same is not true in the complete noncompact setting, even when the space is a Riemannian manifold:

Example 4.1 The cylindrical cusp manifold

$$
M=\mathbb{R} \times e^{r} \mathbb{S}^{1}
$$

has $\tilde{M}^{\delta}=M$ for all $\delta$ while the universal cover is diffeomorphic to a plane.

In this section we investigate the relationship between the delta covers and the universal covers of a complete noncompact manifold. Note that in 35 we used relative $\delta$ covers of balls to prove existence of universal covers in some noncompact settings, but here we are focusing on the delta covers of the manifold itself.

\subsection{The fundamental group and the universal slipping group}

We begin by examining the elements of the fundamental group which cause the problem seen in Example 4.1

Definition 4.1 For each element $g \in \pi_{1}(M), p \in M$, let $L(g, p)=d_{\tilde{M}}(\tilde{p}, g \tilde{p})$, where $\tilde{p}$ is some lift of $p$ in the universal cover $\tilde{M}$, i.e. the length of a shortest representative of $g$ at $p$.

Definition 4.2 The slipping elements $g$ in the fundamental group such that

$$
L(g)=\inf _{x \in \tilde{M}} d_{\tilde{M}}(x, g x)=0
$$

The loops representing these elements slide out to infinity and their lengths disappear.

The set of slipping elements is not a group since it need not be closed as we will see in Example 4.3 . However, it generates a group we call the slipping group. 
Example 4.2 The slipping group of the isometric product cylinder $\mathbb{R} \times_{1} \mathbb{S}^{1}$ is empty. The slipping group of a warped product cylinder $\mathbb{R} \times_{f} \mathbb{S}^{1}$ where $\lim _{r \rightarrow \infty} f(r)=0$ is the entire fundamental group, $\mathbb{Z}$. The slipping group of the doubly warped product $\mathbb{R} \times_{f} \mathbb{S}^{1} \times_{h} \mathbb{S}^{1}$ where $f$ is uniformly bounded below but $\lim _{r \rightarrow \infty} h(r)=0$ is $\mathbb{Z}$ which is a subgroup of $\pi_{1}=\mathbb{Z} \times \mathbb{Z}$.

Of course in these examples the slipping elements and the slipping group agree. Next we see why we need to generate the slipping group.

Example 4.3 We begin by taking a figure 8 formed by taking two circle of radius 1 joined at a point. Let $M^{2}$ be a smooth surface of genus 2 which is obtained by taking the boundary of the tubular neighborhood of radius $1 / 2$ about this figure eight in $\mathbb{R}^{3}$ and smoothing it slightly. Cross $M^{2}$ with a line. For the negative direction on the line, $r \in(-\infty, 1]$, take the isometric product metric. For the positive direction, $r \in[1, \infty)$, of the line, change the metric on $M^{2}$ smoothly so that it is the smoothened tubular neighborhood of radius $1-1 /(2 r)$ about the fixed figure eight.

Now the fundamental group of the figure eight (and $M^{2} \times \mathbb{R}$ ) is the free group on two elements $g_{1}$ and $g_{2}$ where $g_{1}$ goes once around the first circle and $g_{2}$ goes once around the second. The slipping elements of $M^{2} \times \mathbb{R}$ with this metric, are $g_{1}^{j}$ and $g_{2}^{j}$ where $j \in \mathbb{Z}$. The slipping group is the whole fundamental group. However $g_{1} g_{2}$ and other mixed elements are not slipping elements, because in order to go around both holes, they must have length $\geq 2$.

There is a possibly larger group we can call the universal slipping group which is defined as follows:

Definition 4.3 An element $g$ in the fundamental group is in the universal slipping group of $M$, denoted $\pi_{\text {slip }}(M)$, if for all $\delta>0$ there exist elements $g_{1}, g_{2}, \ldots g_{N} \subset \pi_{1}(M)$ such that $L\left(g_{j}\right)<\delta$ and $g=g_{1} g_{2} \cdots g_{N}$. Here $N$ may depend on $\delta$ and on $g$.

Clearly the slipping group is a subgroup of the universal slipping group. The universal slipping group may be strictly larger than the slipping group as can be seen in the following example:

Example 4.4 We construct a complete noncompact surface, $M^{2}$, as follows. First we take the "pair of pants", $P^{2}$, and endow it with a Riemannian metric so that the region near the "waist" of the pants, is isometric to a cylinder $S_{2 \pi}^{1} \times[0, \varepsilon)$ and the regions near the legs are isometric to cylinders $S_{\pi}^{1} \times[0, \varepsilon)$ where $\mathbb{S}_{r}^{1}$ is a circle of intrinsic diameter $r$. We further require that the shortest loop homotopic to the waist in $P^{2}$ has length $4 \pi$ and the shortest loop homotopic to a leg in $P^{2}$ has length $2 \pi$. We glue together two pairs of pants at the waist and call the waist where they are glued $\gamma_{1}$. Then we glue on four more pairs of pants (rescaled by $1 / 2$ ) so that their waists are glued into the four legs along $\gamma_{2,1}, \gamma_{2,2}, \gamma_{2,3}, \gamma_{2,4}$. Next we glue eight more pairs of pants (rescaled by $1 / 4)$ so that their waists are glued to the eight legs along $\gamma_{3,1}, \ldots \gamma_{3,8}$ and so on ad infinitum. This forms a complete noncompact metric space with a collection of closed geodesics $\gamma_{i, j}$ where $i \in \mathbb{N}$ and $j \in 1, \cdots, 2^{i}$. These geodesics are the shortest curves in their free homotopy classes. If we set $\sigma_{i, j}$ to be a minimizing geodesic from $\gamma_{1}(0)$ to $\gamma_{i, j}(0)$, then we have

$$
g_{i, j}=\left[\sigma_{i, j}^{-1} * \gamma_{i, j} * \sigma_{i, j}\right] \in \pi_{1}\left(M^{2}\right)
$$

such that $L\left(g_{i, j}\right)=L\left(\gamma_{i, j}\right)=(1 / 2)^{i} 2 \pi$ and (assuming they are oriented and ordered consistently)

$$
g_{i, j}=g_{i+1,2 j-1} g_{i+1,2 j}
$$

Thus every element of $\pi_{1}\left(M^{2}\right)$ is in the universal slipping group although no element of $\pi_{1}\left(M^{2}\right)$ is in the slipping group.

We have the following nice description for the universal slipping group: 
Lemma 4.4 When viewed as subsets of $\pi_{1}(M)$ :

$$
\pi_{s l i p}(M)=\bigcap_{\delta>0} \pi_{1}\left(\tilde{M}^{\delta}\right)
$$

Proof: Let $g \in \bigcap_{\delta>0} \pi_{1}\left(\tilde{M}^{\delta}\right)$, then for all $\delta>0 g \in \pi_{1}\left(\tilde{M}^{\delta}\right)$. By the definition of $\pi_{1}(M, \delta)$ in (2.6), we know there exists elements of length $<2 \delta$ whose product is $g$, and since this is for all $\delta$, $\mathrm{g}$ is in the slipping group.

Assume on the other hand that $\mathrm{g}$ is in the slipping group, then for all $\delta>0, g$ is a product of elements of length less than $\delta$ so it is in $\pi_{1}(M, \delta / 2)$.

\subsection{The universal cover and the universal $\delta$ cover}

On a compact length space with a universal cover we proved in [36] that the covering spectrum is finite and that the universal cover is the $\delta$ cover corresponding to the smallest $\delta$ in the spectrum. On a complete noncompact length space with universal cover, clearly the covering spectrum may have infinitely many elements:

Example 4.5 A surface of infinite genus with the same size holes has a finite covering spectrum. However, if the size of the holes goes to zero as they approach to infinity, then the surface has a covering spectrum consisting of infinitely many elements whose infimum is 0 and the universal cover is not a $\delta$ cover.

Note that in this example the universal cover is not a delta cover but it is a limit of the $\delta$ covers as $\delta \rightarrow 0$. That is, $\tilde{M}$ is the pointed Gromov Hausdorff limit of $\tilde{M}^{\delta}$ as $\delta \rightarrow 0$ where the points at the centers of the balls used for this limit are the lifts of a fixed point in the space.

We introduce the universal delta cover and prove that it can always be obtained as such a pointed Gromov-Hausdorff limit:

Definition 4.5 The universal delta cover, $\tilde{M}^{0}$, of a length space, $M$, is a covering space which covers all delta covers, $\tilde{M}^{\delta}$. We require further that for any other covering space $\tilde{M}^{\prime}$ which covers all $\delta$ covers, $\tilde{M}^{\delta}$, we have $\tilde{M}^{\prime}$ covers $\tilde{M}^{0}$.

Theorem 4.6 The pointed Gromov-Hausdorff limit,

$$
\lim _{\delta \rightarrow 0} \tilde{M}^{\delta}
$$

of a sequence of $\delta$ covers of a fixed complete length space with universal cover, $M$, based at the lifts of a fixed point $p \in M$ exists and is the universal $\delta$ cover $\tilde{M}^{0}$. Thus it doesn't depend on the basepoint used to define the pointed Gromov-Hausdorff limit.

Proof: First we will just show that for any $p$ and any sequence $\delta_{j} \rightarrow 0$, there is a converging subsequence which converges to a cover. To get uniqueness, we will show it is the universal delta cover in the sense described.

Let $f_{j}: \tilde{M}^{\delta_{j}} \rightarrow M$ be the covering maps so that $f\left(p_{j}\right)=p$. Let $h_{j}: \tilde{M} \rightarrow \tilde{M}^{\delta_{j}}$ be covering maps so that $f(\tilde{p})=p_{j}$. For any fixed $R, \epsilon>0$ let $N(\epsilon, R)$ be the maximum number of disjoint balls of radius $\epsilon$ in $B_{\tilde{p}}(R) \subset \tilde{M}$. This provides a uniform upper bound on the number of disjoint balls of radius $\epsilon$ in $B_{p_{j}}(R) \subset \tilde{M}^{\delta_{j}}$. Thus by Gromov's compactness theorem a subsequence of the $B_{p_{j}}(R)$ converges. By Grove Petersen's Arzela Ascoli theorem and the fact that $f_{j}$ and $h_{j}$ are distance nonincreasing maps, subsequences of these functions converge as well. Taking $R \rightarrow \infty$ and diagonalizing we get a limit space $\tilde{M}^{0}$ with distance nonincreasing maps $h_{\infty}: \tilde{M} \rightarrow \tilde{M}^{0}$ and 
$f_{\infty}: \tilde{M}^{0} \rightarrow M$ such that the concatenation of these functions is a covering map. Thus $f_{\infty}$ must be a covering map as well since it will act as an isometry on any ball lifted isometrically to the universal cover.

Now this covering space we have obtained (which may depend on the subsequence $\delta_{j}$ and on the point $p$ ), covers all the delta covers of the space. This can be seen, by fixing $\delta>0$ and observing that eventually $\delta_{j}<\delta$, so it is a limit of spaces which cover $\tilde{M}^{\delta}$, and thus the same arguement used above to explain why it covers $M$ can be used to explain why it covers $\tilde{M}^{\delta_{j}}$.

Furthemore if $N$ covers all of $\tilde{M}^{\delta}$, then $N$ covers all the $\tilde{M}^{\delta_{j}}$ and, as argued above these covering maps have a convergence subsequence to a covering map from $N$ to $\tilde{M}^{0}$.

Thus $\tilde{M}^{0}$ is the universal $\delta$ cover and so it is unique and doesn't depend on the choice of $p$ or the subsequence, and so no subsequence was required for the limit after all.

We can now extend Theorem 3.4 of [36]

Proposition 4.7 If the infimum of the covering spectrum is positive then the universal delta cover is a delta cover and the infimum is in the covering spectrum.

Proof: If the spectrum has a positive infimum $\inf \{\delta\}$, let $\delta_{0}=\inf \{\delta\} / 2$. Then the $\delta_{0}$ cover will cover all $\delta$ covers and so it must be the universal delta cover by the uniqueness in Theorem 4.6. Now there exists $\delta_{i}$ in the covering spectrum decreasing to $\inf \{\delta\}$, and so by Defn 2.5, we have

$$
\tilde{X}^{\delta_{i}} \neq \tilde{X}^{\delta^{\prime}} \quad \forall \delta^{\prime}>\delta_{i} .
$$

By the monotonicity of $\delta$ covers, the $\inf \{\delta\}$ cover must cover these $\delta_{i}$ covers and so for every $i$ we have

$$
\tilde{X}^{\inf \{\delta\}} \neq \tilde{X}^{\delta^{\prime}} \quad \forall \delta^{\prime}>\delta_{i} .
$$

Taking $\delta_{i}$ down to $\inf \{\delta\}$ we see that $\inf \{\delta\}$ satisfies the requirements of Defn 2.5.

Remark 4.8 In general, however, the universal delta cover need not be a delta cover. This can be seen in Example 4.5, where each $\delta$ cover unravels finitely many holes and the universal delta cover unwraps all of them.

Remark 4.9 The universal delta cover need not be the universal cover either. In the cusp cylinder of Example 4.1. $M$, all the delta covers, $\tilde{M}^{\delta}$, are just isometric to $M$ and thus so is their GromovHausdorff limit, the universal delta cover. Observe that in this example the universal slipping group $\pi_{\text {slip }}$, is the whole fundamental group.

Theorem 4.10 Given a complete length space, $M$, with a universal cover, $\tilde{M}$, fundamental group $\pi_{1}(M)$, and universal slipping group $\pi_{\text {slip }}(M) \subset \pi_{1}(M)$. Then the universal delta covering space $\tilde{M}^{0}$ satisfies

$$
\tilde{M}^{0}=\tilde{M} / \pi_{\text {slip }}(M)
$$

and is thus a regular covering space.

Proof: From Lemma 4.4, it is enough to show

$$
\tilde{M}^{0}=\tilde{M} /\left(\bigcap_{\delta>0} \pi_{1}(M, \delta)\right) .
$$

This follows immediately since $\tilde{M} /\left(\bigcap_{\delta>0} \pi_{1}(M, \delta)\right)$ covers all $\delta$-cover $\tilde{M}^{\delta}$ and any cover covers all the $\delta$-cover covers $\tilde{M} /\left(\bigcap_{\delta>0} \pi_{1}(M, \delta)\right)$. Therefore $\tilde{M} /\left(\bigcap_{\delta>0} \pi_{1}(M, \delta)\right)$ is the universal delta-cover $\tilde{M}^{0}$. 
Remark 4.11 In Example 4.4, the universal slipping group of the surface, $M^{2}$, was the whole fundamental group. So the universal delta cover of $M^{2}$ is just $M^{2}$. Thus all delta covers of this space are just $M^{2}$.

An immediate consequence of Theorem 4.10 combined with Proposition 4.7 is:

Corollary 4.12 If the slipping group is empty and the covering spectrum has a positive infimum then the universal cover is a $\delta$ cover.

Remark 4.13 If one extends the notion of slipping group and universal delta cover to complete length spaces which are not known to have a universal cover, a theorem similar to Corollary 4.12 might be applied to prove the existence of a universal cover.

\section{The Rescaled Covering Spectra}

In this section, we define two scale invariant spectra called the Rescaled Covering Spectrum and the Rescaled Covering Spectrum at Infinity. The first of these will be defined for pointed spaces $(X, x)$ and the latter will not depend on a basepoint. Both will be invariant when the space is rescaled.

Unlike the ordinary covering spectrum, the rescaled spectra will be initially defined without covering spaces. Instead we will assume the spaces have universal covers and use subgroups of the fundamental group to define the spectra just as the covering spectrum may be computed using (2.8). At the end of this section we propose a possible means of extending the definition to spaces without universal covers [Remark 5.15]. However we believe the main applications of these spectra are to submanifolds of Euclidean space and to Riemannian manifolds both of which always have universal covers.

\subsection{The rescaled length and the infinite rescaled length}

We begin with the rescaled length:

Definition 5.1 Given a pointed space $\left(X, x_{0}\right)$ with a universal cover, we defined the rescaled length of an element $g \in \pi_{1}(X)$ envisioning it as a deck transform:

$$
L_{r s}^{x_{0}}(g)=\inf _{x \in X \backslash\left\{x_{0}\right\}} \frac{d_{\tilde{X}}(g \tilde{x}, \tilde{x})}{d_{X}\left(x, x_{0}\right)} .
$$

where $\tilde{x}$ is a lift of $x$ to the universal cover.

By definition this is scale invariant, so if we rescale the metric on our space, we get the same rescaled lengths for all $g$.

Remark 5.2 As in Remark 3.5, one might try to extend this definition to spaces without universal covers. Here, however, we will keep things simple.

Note that for a standard cylinder the rescaled length of all elements are zero, while for the one sheeted hyperboloid, $\left\{(x, y, z): x^{2}+y^{2}=z^{2}+1\right\}$, the rescaled length of the generator is 2 . See Example 6.1 for more details. In both of these examples the infimum is not achieved.

However, for some spaces, the rescaled length is achieved and is highly dependant on the basepoint. This occurs with spaces like the catenoid and those with handles where for most points in the manifold the shortest geodesic based at a point must traverse all the way into some central location and then go all the way back out: 
Example 5.1 Let $X$ be a handlebody with a cusp and $g$ an element with length, $L(g)<2$, represented by a loop running around the handle. Suppose $\sigma$ is the minimal loop running around the handle so $L(\sigma)=L(g)$. For $x$ far from the handle, a loop freely homotopic to $\sigma$ based at $x$ would have to traverse all the way to the loop and then come back so

$$
\frac{d_{\tilde{X}}(\tilde{x}, g \tilde{x})}{d_{X}(x, \sigma)} \rightarrow 2
$$

No matter where $x_{0}$ is located

$$
\lim _{x \rightarrow \infty} \frac{d_{\tilde{X}}(\tilde{x}, g \tilde{x})}{d_{X}\left(x, x_{0}\right)} \rightarrow 2 .
$$

On the other hand if we choose $x$ on $\sigma$, we see that

$$
L_{r s}^{x_{0}}(g) \leq \frac{d_{\tilde{X}}(g \sigma(t), \sigma(t))}{d_{X}\left(\sigma(t), x_{0}\right)} \leq \frac{L(\sigma)}{\inf _{t \in \mathbb{S}^{1}} d_{X}\left(\sigma(t), x_{0}\right)} .
$$

So if we choose a basepoint $x_{0}$ sufficiently far from $\sigma$ we get

$$
L_{r s}^{x_{0}}(g)<2
$$

So $L_{r s}^{x_{0}}(g)$ is achieved at some point $x$ rather than approached as $x \rightarrow \infty$. If we fix a particular such $x_{0}$ we get some positive value $L_{r s}^{x_{0}}(g)=f\left(x_{0}\right)>0$.

However this function $f$ is highly dependant on the basepoint $x_{0}$ and in fact decays to 0 as we choose $x_{0}$ further and further from $\sigma$ :

$$
f\left(x_{0}\right) \leq \frac{d_{\tilde{X}}(g \sigma \tilde{(t)}, \tilde{\sigma(t))}}{d_{X}\left(\sigma(t), x_{0}\right)} \leq \frac{L(\sigma)}{d_{X}\left(\sigma(t), x_{0}\right)} .
$$

In our next definition, we define a scale invariant length which we will prove does not depend on a basepoint in Lemma 5.4 below:

Definition 5.3 Given a complete length space $X$ with a universal cover the infinite rescaled length of an element of the deck transforms is:

$$
L_{r s}^{\infty}(g)=\lim _{R \rightarrow \infty} \inf _{x \in X \backslash B_{x_{0}}(R)} \frac{d_{\tilde{X}}(g \tilde{x}, \tilde{x})}{d_{X}\left(x, x_{0}\right)} .
$$

where $\tilde{x}$ is a lift of $x$ to the universal cover and $x_{0} \in X$.

Here the $r s$ in $L_{r s}^{x_{0}}$ refer to the word "rescaled". They are not parameters.

Lemma 5.4 The infinite rescaled length of a deck transform does not depend on the basepoint $x_{0}$ used in (5.42).

Proof: Note that there exists $x_{i} \rightarrow \infty$ such that

$$
L_{r s}^{\infty}\left(g, x_{0}\right)=\lim _{i \rightarrow \infty} \frac{d_{\tilde{M}}\left(g \tilde{x}_{i}, \tilde{x}_{i}\right)}{d\left(x_{i}, x_{0}\right)} .
$$

Let $d\left(x_{0}, y_{0}\right)=r$. Then by the triangle inequality,

$$
\frac{d_{\tilde{M}}\left(x_{i}, g x_{i}\right)}{d\left(x, y_{0}\right)} \geq \frac{d_{\tilde{M}}\left(x_{i}, g x_{i}\right)}{d\left(x, x_{0}\right)+r} .
$$

Taking $x_{i}$ to infinity we get:

$$
L_{r s}^{\infty}\left(g, y_{0}\right) \leq \lim _{i \rightarrow \infty} \frac{d_{\tilde{M}}\left(g \tilde{x}_{i}, \tilde{x}_{i}\right)}{d\left(x_{i}, y_{0}\right)} \leq L_{r s}^{\infty}\left(g, x_{0}\right) .
$$


Lemma 5.5 Given $X$ and choosing any basepoint $x_{0}$ to define $L_{r s}^{x_{0}}$ we have

$$
L_{r s}^{\infty}(g) \geq L_{r s}^{x_{0}}(g) .
$$

Proof: This is just a matter of noting that the the infimum in the definition of $L_{r s}^{\infty}$ is over a smaller set than the one in the definition $L_{r s}^{x_{0}}$.

Lemma 5.6 The rescaled length and infinite rescaled lengths are always $\leq 2$.

Proof: Let $\left(X, x_{0}\right)$ be a complete length space with a universal cover and $g \in \pi_{1}(X)$. Let $\gamma$ run between $\tilde{x}_{0}$ and $g \tilde{x}_{0}$ in $\tilde{X}$. Then for any sequence $x_{i} \rightarrow \infty$ we have

$$
\begin{aligned}
L_{r s}^{\infty}(g) & \leq \lim _{i \rightarrow \infty} \frac{d_{\tilde{M}}\left(g \tilde{x}_{i}, \tilde{x}_{i}\right)}{d\left(x_{i}, x_{0}\right)} \\
& \leq \lim _{i \rightarrow \infty} \frac{2 d_{\tilde{M}}\left(\tilde{x}_{i}, \tilde{x}_{0}\right)+L(\gamma)}{d\left(x_{i}, x_{0}\right)}=2
\end{aligned}
$$

Lemma 5.7 For a given element $g \in \pi_{1}(X), L_{r s}^{x_{0}}(g)=0$ iff $L_{r s}^{\infty}(g)=0$.

Proof: If $L_{r s}^{\infty}(g)=0$, then there exists $x_{i} \rightarrow \infty$ such that

$$
\lim _{i \rightarrow \infty} d\left(\tilde{x}_{i}, g \tilde{x}_{i}\right) / d\left(x_{0}, x_{i}\right)=0
$$

so the infimum in the definition of $L_{r s}^{x_{0}}(g)$ is 0 as well. We get the converse using (5.5).

\subsection{Rescaled groups and rescaled delta covers}

Definition 5.8 The rescaled slipping group is the group generated by $g \in \pi_{1}(X)$ with $L_{r s}^{\infty}(g)=0$ and is denoted

$$
\pi_{r s}^{\infty}(X, 0)
$$

Definition 5.9 The rescaled $\delta$ covering group, $\pi_{r s}^{x_{0}}(X, \delta)$, of a pointed complete length space $\left(X, x_{0}\right)$ with a universal cover is the subgroup of $\pi_{1}(X)$ generated by elements of rescaled length $L_{r s}^{x_{0}}(g)<2 \delta$ :

$$
\pi_{r s}^{x_{0}}(X, \delta)=\left\langle g: L_{r s}^{x_{0}}(g)<2 \delta\right\rangle \subset \pi_{1}(X)
$$

Similarly the infinitely rescaled $\delta$ covering group is

$$
\pi_{r s}^{\infty}(X, \delta)=\left\langle g: L_{r s}^{\infty}(g)<2 \delta\right\rangle \subset \pi_{1}(X)
$$

These groups are scale invariant:

$$
\pi_{r s}^{x_{0}}(X, \delta)=\pi_{r s}^{x_{0}}(X / R, \delta) \text { and } \pi_{r s}^{\infty}(X, \delta)=\pi_{r s}^{\infty}(X / R, \delta) .
$$

Definition 5.10 A rescaled $\delta$ cover of a space $X$ with a universal cover is

$$
\tilde{X}_{r s}^{\delta, x_{0}} / \pi_{r s}^{x_{0}}(X, \delta)
$$

and the infinite rescaled delta cover is

$$
\tilde{X}_{r s}^{\delta, \infty} / \pi_{r s}^{\infty}(X, \delta)
$$


Lemma 5.11 We have

$$
\pi_{r s}^{\infty}(X, \delta) \subset \pi_{r s}^{x_{0}}(X, \delta)
$$

Thus

$$
\tilde{X}_{r s}^{\delta, x_{0}} \text { covers } \tilde{X}_{r s}^{\delta, \infty} .
$$

When $\delta>1$, the group is the entire fundamental group and the covering spaces are just the original space $X$.

Proof: Given $g \in \pi_{r s}^{\infty}(X, \delta), L_{r s}^{\infty}(g) \leq 2 \delta$, so by (5.5),$L_{r s}^{x_{0}}(g) \leq 2 \delta$, and $g \in \pi_{r s}^{x_{0}}(X, \delta)$. Lemma 5.6 justifies the claims regarding $\delta=1$.

\subsection{Rescaled covering spectra defined}

Definition 5.12 Given a pointed complete length space $\left(X, x_{0}\right)$, with a universal cover, $\tilde{X}$, the rescaled covering spectrum of $X$ denoted $\operatorname{CovSpec} r_{r s}^{x_{0}}(X)$, is the set of all $\delta>0$ such that

$$
\pi_{r s}^{x_{0}}(X, \delta) \neq \pi_{r s}^{x_{0}}\left(X, \delta^{\prime}\right) \quad \forall \delta^{\prime}>\delta
$$

when viewed as subsets of $\pi_{1}(X)$. The infinite rescaled covering spectrum, CovSpec ${ }_{r s}^{\infty}(X)$, is defined similarly as the set of all $\delta>0$ such that

$$
\pi_{r s}^{\infty}(X, \delta) \neq \pi_{r s}^{\infty}\left(X, \delta^{\prime}\right) \quad \forall \delta^{\prime}>\delta
$$

so that it does not depend on the basepoint $x_{0}$.

By (5.53), we see that these covering spectra are scale invariant:

$$
\operatorname{CovSpec}_{r s}^{x_{0}}(X)=\operatorname{CovSpec}_{r s}^{x_{0}}(X / R) \text { and } \operatorname{CovSpec}_{r s}^{\infty}(X)=\operatorname{CovSpec}_{r s}^{\infty}(X / R)
$$

Proposition 5.13 Given a pointed complete length space $\left(X, x_{0}\right)$, with a universal cover, $\tilde{X}$,

$$
\begin{array}{lll}
\operatorname{CovSpec}_{r s}^{x_{0}}(X) & \subset & (0,1] \\
\operatorname{CovSpec}_{r s}^{\infty}(X) & \subset & (0,1] .
\end{array}
$$

Proof: If $\delta>0 \in \operatorname{CovSpec}_{r s}^{x_{0}}(X)$ then there exists $g_{j} \in \pi_{1}(X) \backslash \pi_{r s}^{x_{0}}(X, \delta)$ such that

$$
\limsup _{j \rightarrow \infty} L_{r s}^{x_{0}}\left(g_{j}\right) / 2=\delta .
$$

By Lemma 5.6 we know $L_{r s}^{\infty}\left(g_{j}\right) \leq 2$. The proof for infinite rescaled cover spectrum is the same.

Remark 5.14 By the theorem in the appendix of [37] it is easy to see the rescaled and infinite rescaled covering spectra are lower semiclosed sets.

\subsection{Further directions}

Remark 5.15 One should be able to extend the notion of the rescaled covering spectra to complete noncompact length spaces without universal covers by adapting the definition of the rescaled lengths as discussed in Remark 5.2.

Remark 5.16 An alternative way of extending the definition of the rescaled covering spectra to spaces without universal covers would be to describe the related covering spaces as Spanier covers with some well chosen sets. One cannot just choose balls $B_{x}\left(r_{x}\right)$ where $r_{x}=\delta d\left(x, x_{0}\right)$. as there is a difficulty with the point $x_{0}$ itself. If one uses an arbitrary ball around $x_{0}$ we lose scale invariance. One possibility might be to take a limit of covers where the radius of the ball about $x_{0}$ is taken to 0.

Remark 5.17 To define the infinite rescaled covering spectra on spaces without universal covers, one needs to figure out how to use open sets to force the length out to infinity. 


\section{Asymptotic Behavior and the Rescaled Covering Spectrum}

Here we study the asymptotic behavior of complete noncompact metric spaces using the rescaled covering spectra and the rescaled slipping group. The first subsection concerns the loops to infinity property and the relationship between the rescaled covering spectra and the cut off covering spectrum defined by the authors in [37. The second subsection explicitly computes the rescaled covering spectra of spectific spaces including hyperboloids and doubly warped products. The final subsection proposes a conjecture concerning the relationship between the diameter growth of a space and its rescaled covering spectrum.

\subsection{Loops to infinity}

We recall the loops to infinity property defined in [32]:

Definition 6.1 Given a metric space, $X$, a loop $\gamma: \mathbb{S}^{1} \rightarrow X$ is said to have the loops to infinity property, if for every compact set $K \subset X$, there is another loop $\sigma: \mathbb{S}^{1} \rightarrow X \backslash K$ freely homotopic to $\gamma$.

Lemma 6.2. If $L_{r s}^{\infty}(g)<2$ then any curve representing $g$ has the loops to infinity property.

Proof: If $g$ does not have the loops to infinity property, its representative curves $C_{i}$ whose lengths approach $L_{r s}^{\infty}(g)$ and have $C_{i}(0) \rightarrow \infty$ must pass through a common compact set, $K$. Thus $L\left(C_{i}\right) \geq$ $2 d_{X}\left(C_{i}(0), K\right)$, so the limit in the definition of rescaled length gives a 2 .

Theorem 6.3 Given a complete length space $X$, with a universal cover, $\tilde{X}$, if

$$
\operatorname{CovSpec}_{r s}^{\infty}(X) \in(0,1)
$$

then

$$
\operatorname{CovSpec}_{\text {cut }}(X)=\emptyset .
$$

This theorem captures the fact that the rescaled covering spectrum and the cut off spectrum measure very different kinds of "holes" in a complete length space.

Proof: By the hypothesis we have

$$
\pi_{r s}^{\infty}(X, 1)=\pi_{r s}^{\infty}\left(X, \delta^{\prime}\right) \quad \forall \delta^{\prime}>1 .
$$

So for any $g_{0} \in \pi_{1}(M)$, taking $\delta^{\prime}>L\left(g_{0}\right) / 2$, we see that $g_{0} \in \pi_{r s}^{\infty}(X, 1)$. So the fundamental group is generated by elements $g$ with $L_{r s}^{x_{0}}(g)<2$. Thus by Lemma 6.2 any curve representing such a generator has the loops to infinity property and so by Theorem 4.20 in [37] $\operatorname{CovSpec}_{\text {cut }}(X)=\emptyset$.

\subsection{Cones, hyperboloids and warped products}

Definition 6.4 The base point free cone over $Y$ with scaling $k$ denoted $C_{k}(Y)$ is

$$
C_{k}(Y)=(0, \infty)_{f} \times Y
$$

where $f(r)=k r$. 
Theorem 6.5 Given a compact length space $Y$, we have

$$
\operatorname{CovSpec}_{r s}^{\infty}\left(C_{k}(Y)\right)=\{(1 / 2) \sqrt{2-2 \cos (\min \{\pi, 2 k \delta\})}: \delta \in \operatorname{Cov} \operatorname{Spec}(Y)\} .
$$

and

$$
\operatorname{CovSpec}_{r s}^{x_{0}}\left(C_{k}(Y)\right)=\emptyset
$$

Proof: Recall that with a linear warped product,

$$
d_{C_{k}(Y)}\left(\left(y_{1}, r_{1}\right),\left(y_{2}, r_{2}\right)\right)=\sqrt{r_{1}^{2}+r_{2}^{2}-2 r_{1} r_{2} \cos \left(\min \left\{\pi, k d_{Y}\left(y_{1}, y_{2}\right)\right\}\right)} .
$$

This can be seen because if $k d_{Y}\left(y_{1}, y_{2}\right)<\pi$ then the minimal geodesic between them has length $<\pi$, and the minimal geodesic between $\left(y_{i}, r_{i}\right)$ lies in a linear warped product of that geodesic (which is a sector of Euclidean space and one can compute its length using the law of cosines. If $k d_{Y}\left(y_{1}, y_{2}\right) \geq \pi$ then the shortest geodesic in the cone passes though the base point and has length

$$
r_{1}+r_{2}=\sqrt{r_{1}^{2}+r_{2}^{2}-2 r_{1} r_{2} \cos (\pi)}
$$

Let $N=C_{k}(Y)$ and $\tilde{N}$ its universal cover. By the definition of the base point free cone, any $g \in \pi_{1}(N)$ is also a $g \in \pi_{1}(Y)$. In fact, one can easily see that

$$
\tilde{N}=(0, \infty) \times_{f} \tilde{Y}=C_{k}(\tilde{Y}),
$$

where any point in $N$ can be represented as $x=(y, r)$ with $x \in Y$ and this point lifts to a point $\tilde{x}=(\tilde{y}, r) \in \tilde{N}$ where $\tilde{y} \in \tilde{Y}$ and where $g(\tilde{x}, r)=(g \tilde{x}, r)$.

Let $y_{1}$ achieve the infimum in the definition of $L(g)$ viewing $g \in \pi_{1}(Y)$ :

$$
L(g)=d_{\tilde{Y}}\left(g \tilde{y_{1}}, \tilde{y_{1}}\right)
$$

and let $y_{0}$ be furthest from $y_{1}$ :

$$
d_{Y}\left(y_{0}, y_{1}\right)=\sup _{y \in Y} d_{Y}\left(y_{1}, y\right)
$$

For any $r_{0}>0$ we take $x_{0}=\left(y_{0}, r_{0}\right)$. We have

$$
r^{-1}(0, R) \subset B_{x_{0}}\left(R+r_{0}\right) \subset r^{-1}\left(0, R+2 r_{0}\right) .
$$

Applying Definition 5.1 which does not depend on the choice of $x_{0}$, we have

$$
\begin{aligned}
L_{r s}^{\infty}(g) & =\lim _{R \rightarrow \infty} \inf _{(y, r) \in N \backslash B_{x_{0}}(R)} \frac{d_{\tilde{N}}(g(\tilde{y}, r),(\tilde{y}, r))}{d_{N}\left((y, r),\left(y_{0}, r_{0}\right)\right)} \\
& =\lim _{R \rightarrow \infty} \inf _{y \in N, r \geq R} \frac{d_{\tilde{N}}((g \tilde{y}, r),(\tilde{y}, r))}{d_{N}\left((y, r),\left(y_{0}, r_{0}\right)\right)} \\
& =\liminf _{r \rightarrow \infty} \inf _{y \in N} \frac{r \sqrt{2-2 \cos \left(\min \left\{\pi, k d_{\tilde{Y}}(g \tilde{y}, \tilde{y})\right\}\right)}}{\sqrt{r^{2}+r_{0}^{2}-2 r r_{0} \cos \left(\min \left\{\pi, k d_{Y}\left(y, y_{0}\right)\right\}\right)}} \\
& =\liminf _{r \rightarrow \infty} \frac{r \sqrt{2-2 \cos \left(\min \left\{\pi, k d_{\tilde{Y}}\left(g \tilde{y}_{1}, \tilde{y}_{1}\right)\right\}\right)}}{\sqrt{r^{2}+r_{0}^{2}-2 r r_{0} \cos \left(\min \left\{\pi, k d_{Y}\left(y_{1}, y_{0}\right)\right\}\right)}} \\
& =\sqrt{2-2 \cos \left(\min \left\{\pi, k d_{\tilde{Y}}\left(g \tilde{y}_{1}, \tilde{y}_{1}\right)\right\}\right)} \\
& =\sqrt{2-2 \cos (\min \{\pi, k L(g)\})}
\end{aligned}
$$


This implies the first claim in the statement of our theorem.

In contrast, when we compute the rescaled length depending on a given basepoint $x_{0}=\left(y_{0}, r_{0}\right)$, we choose any $y_{2} \neq y_{0}$ and we have

$$
\begin{aligned}
L_{r s}^{x_{0}}(g) & =\inf _{(y, r) \neq\left(y_{0}, r_{0}\right)} \frac{d_{\tilde{N}}(g(\tilde{y}, r),(\tilde{y}, r))}{d_{N}\left((y, r),\left(y_{0}, r_{0}\right)\right)} \\
& =\inf _{(y, r) \neq\left(y_{0}, r_{0}\right)} \frac{r \sqrt{2-2 \cos \left(\min \left\{\pi, k d_{\tilde{Y}}(g \tilde{y}, \tilde{y})\right\}\right)}}{\sqrt{r^{2}+r_{0}^{2}-2 r r_{0} \cos \left(\min \left\{\pi, k d_{Y}\left(y, y_{0}\right)\right\}\right)}} \\
& \leq \inf _{r \neq r_{0}} \frac{r \sqrt{2-2 \cos \left(\min \left\{\pi, k d_{\tilde{Y}}\left(g \tilde{y}_{2}, \tilde{y}_{2}\right)\right\}\right)}}{\sqrt{r^{2}+r_{0}^{2}-2 r r_{0} \cos \left(\min \left\{\pi, k d_{Y}\left(y_{2}, y_{0}\right)\right\}\right)}}=0 .
\end{aligned}
$$

This effect where the rescaled spectrum goes to 0 does not appear to happen in a setting where the space $N$ is a manifold that is asymptotically cone like. Although the infinite rescaled spectrum appears to behave in the same way. This can be seen in the following example:

Example 6.1 The one-sheeted hyperboloid,

$$
N^{2}=\left\{(x, y, z): x^{2}+y^{2}=z^{2}+1\right\},
$$

has a rescaled covering spectrum which consists of a single value

$$
\begin{aligned}
& \operatorname{CovSpec}_{r s}^{x_{0}}(N)=\{1\} \\
& \operatorname{CovSpec}_{r s}^{\infty}(N)=\{1\} .
\end{aligned}
$$

Proof: To compute $L_{r s}^{x_{0}}(g)$, we take advantage of the invariance under rescaling:

$$
\begin{aligned}
\left\{(x, y, z): x^{2}+y^{2}=z^{2}+1\right\} / R & =\left\{(R x, R y, R z): x^{2}+y^{2}=z^{2}+1\right\} \\
& =\left\{(x, y, z): x^{2}+y^{2}=z^{2}+1 / R^{2}\right\}
\end{aligned}
$$

Let $\sigma \subset N$ be the circular neck. As $R \rightarrow \infty$, we see that the length of a shortest geodesic $\gamma$ based at any point $\gamma(0) \in N \backslash T_{r}(\sigma)$ is approaching the length of such a geodesic in the flat cone with the origin removed:

$$
\left\{(x, y, z): x^{2}+y^{2}=z^{2},(x, y, z) \neq(0,0,0)\right\} .
$$

This is $C_{k}(Y)$ in (6.67) with $k=1, Y$ the circle with radius $1 / \sqrt{2}$. $\operatorname{Covspec}(Y)=\left\{\frac{\pi}{\sqrt{2}}\right\}$, hence Theorem 6.5 gives

$$
\operatorname{CovSpec}_{r s}^{\infty}\left(C_{k}(Y)\right)=\{1\} .
$$

Since it is scale invariant, so $\operatorname{CovSpec}_{r s}^{\infty}\left(N^{2}\right)=\{1\}$.

In the following theorem we see that warped product spaces which are asymptotic to such cones, and even double warped producs which need not be close to the cones but are simply connected on their second warping factor, have the same rescaled covering spectra as their cones.

Theorem 6.6 Suppose our space is a doubly warped product

$$
X=[0, \infty) \times_{f} N \times_{h} M
$$

where $N$ and $M$ are compact Riemannian manifolds with $f^{\prime}(0)=0, f(0)>0$ and either $h^{\prime}(0)=0$ or $h(0)=0$. In all these cases we assume $f(r)>0$ and $h(r)>0$ on $(0, \infty)$. 
If $M$ is simply connected or $h(0)=0$ we have

$$
L_{r s}^{\infty}(g)=\liminf _{r \rightarrow \infty} \frac{F\left(r, L_{N}(g)\right)}{r} .
$$

where $F(r, d)$ is the length of a minimal geodesic in

$$
\mathbb{R} \times_{f} \mathbb{R}
$$

between $(r, 0)$ and $(r, d)$ and $L_{N}(g)$ is the length of $g$ viewed as an element of $\pi_{1}(N)$.

Proof: Observe that the universal cover

$$
\tilde{X}=[0, \infty) \times_{f} \tilde{N} \times_{h} M
$$

where $x=(r, y, z)$ lifts to $\tilde{x}=(r, \tilde{y}, z)$ and any $g \in \pi_{1}(X)$ can be viewed as $g \in \pi_{1}(N)$ so that

$$
g \tilde{x}=(r, g \tilde{y}, z) .
$$

Also we note that if

$$
X^{\prime}=[0, \infty) \times_{f} N
$$

then $\tilde{X}^{\prime}=[0, \infty) \times_{f} \tilde{N}$ and that

$$
d_{X^{\prime}}\left(\left(r_{1}, y_{1}\right),\left(r_{2}, y_{2}\right)\right) \leq d_{X}\left(\left(r_{1}, y_{1}, z_{1}\right),\left(r_{2}, y_{2}, z_{2}\right)\right) \leq d_{X^{\prime}}\left(\left(r_{1}, y_{1}\right),\left(r_{2}, y_{2}\right)\right)+\max _{\left[r_{1}, r_{2}\right]} h(r) d_{M}\left(z_{1}, z_{2}\right) .
$$

So applying Definition 5.1 with $x_{0}=\left(0, y_{0}, z_{0}\right)$ we have

$$
\begin{aligned}
L_{r s}^{\infty}(g) & =\lim _{R \rightarrow \infty} \inf _{(r, y, z) \in X \backslash B_{R}\left(0, y_{0}, z_{0}\right)} \frac{d_{\tilde{X}}((r, g \tilde{y}, z),(r, \tilde{y}, z))}{d_{X}\left((r, y, z),\left(0, y_{0}, z_{0}\right)\right)} \\
& =\liminf _{r \rightarrow \infty} \inf _{y \in N, z \in M} \frac{d_{\tilde{X}}((r, g \tilde{y}, z),(r, \tilde{y}, z))}{d_{X}((r, y, z),(0, y, z)) \pm d_{X}\left(\left(0, y_{0}, z_{0}\right),(0, y, z)\right)} \\
& =\liminf _{r \rightarrow \infty} \inf _{y \in N, z \in M} \frac{d_{\tilde{X}}((r, g \tilde{y}, z),(r, \tilde{y}, z))}{d_{X}((r, y, z),(0, y, z))} \\
& =\liminf _{r \rightarrow \infty} \inf _{y \in N} \frac{d_{\tilde{X}^{\prime}}((r, g \tilde{y}),(r, \tilde{y}))}{d_{X^{\prime}}((r, y),(0, y))} \\
& =\liminf _{r \rightarrow \infty} \inf _{y \in N} \frac{F\left(r, d_{\tilde{N}}(g \tilde{y}, \tilde{y})\right)}{r} .
\end{aligned}
$$

Observe that $F(r, d)$ is increasing as $d$ increases so

$$
\inf _{y \in N} \frac{F\left(r, d_{\tilde{N}}(g \tilde{y}, \tilde{y})\right)}{r}=\frac{F\left(r, L_{N}(g)\right)}{r}
$$

where $L_{N}(g)$ be the length of $g$ as an element of $\pi_{1}(N)$. Thus

$$
L_{r s}^{\infty}(g)=\liminf _{r \rightarrow \infty} \frac{F\left(r, L_{N}(g)\right)}{r} .
$$


Corollary 6.7 Suppose that

$$
\lim _{r \rightarrow \infty} \frac{f(r)}{r}=k
$$

If $k>0$ we have

$$
\operatorname{CovSpec}_{r s}^{\infty}(X)=\left\{\frac{1}{2} \sqrt{2-2 \cos (\min \{\pi, 2 k d\}): d \in \operatorname{CovSpec}(N)}\right.
$$

and the rescaled slipping group is empty. If $k=0$ then

$$
\operatorname{CovSpec}_{r s}^{\infty}(X)=\emptyset \text {. }
$$

and $\pi_{1}(X) \subset \pi_{r s}^{\infty}(X, 0)$.

Proof: In the special case where

$$
\lim _{r \rightarrow \infty} f(r) / r=0
$$

then

$$
\lim _{r \rightarrow \infty} F(r, d) / r=0
$$

so $\operatorname{CovSpec} \operatorname{sis}_{r s}^{\infty}(X)=\emptyset$ and $\pi_{1}(X)$ is a subset of the rescaled slipping group. Alternately if

$$
\lim _{r \rightarrow \infty} f(r) / r=k>0,
$$

then

$$
\lim _{r \rightarrow \infty} F(r, d) / r=\sqrt{2-2 \cos (\min \{\pi, 2 k d\})}
$$

so we have our claim.

\subsection{Diameter growth}

For any metric space $X, p \in X$,

$$
\limsup _{r \rightarrow \infty} \frac{\operatorname{diam}\left(\partial B_{p}(r)\right)}{r}=a
$$

with $0 \leq a \leq 2$.

Conjecture 6.8 For a metric space $X$ with a above,

$$
\operatorname{CovSpec}_{r s}^{\infty}(X) \subset\left(0, \frac{a}{2}\right] \cup\{1\} .
$$

In particular, when $X$ has sublinear diameter growth, i.e. $a=0$, then

$$
\operatorname{CovSpec}_{r s}^{\infty}(X) \subset\{1\} .
$$

\section{Applications with Curvature Bounds}

In this section we restrict ourselves to the study of complete Riemannian manifolds, $M$. In the first subsection we study manifolds with nonnegative sectional curvature, whose topology can be understood because such manifolds have a compact soul. We also present an interesting example of Wilking which has nonnegative sectional curvature and positive Ricci curvature and prove it has a nontrivial rescaled covering spectrum. In the next subsection we study manifolds with nonnegative Ricci curvature. We first show that the fundamental group of the classic example of Nabonnand lies in the rescaled slipping group [Proposition 7.7. We prove Theorem 1.1 concerning manifolds with positive Ricci curvature and its Corollary 7.5 , We close with a subsection on the rescaled covering spectra of manifolds with nonnegative Ricci curvature proving Theorem 1.2 . 


\subsection{Nonnegative sectional curvature}

Cheeger-Gromoll $[8]$ proved that complete manifolds with nonnegative sectional curvature are diffeomorphic to normal bundles over totally geodesic compact submanifolds called souls. Sharafutdinov [30] then proved there was a distance nonincreasing retraction to the soul: $P: M \rightarrow S$. Perelman [27, 28] showed that $P$ is a Riemannian submersion and extended the distance nonincreasing retraction to complete Alexandrov spaces with nonnegative curvature. In [37] using the distance nonincreasing retraction, $P$, the authors proved that the covering spectrum of these spaces behave exactly like the covering spectrum of a compact space:

Theorem 7.1 37] If $M^{n}$ is a complete noncompact Alexandrov space with nonnegative curvature, then

$$
\operatorname{CovSpec}\left(M^{n}\right)=\operatorname{Cov} \operatorname{Spec}\left(S^{k}\right)
$$

where $S^{k}$ is its soul and

$$
\operatorname{CovSpec}\left(M^{n}\right) \subset(1 / 2) \operatorname{Length}\left(M^{n}\right)=(1 / 2) \operatorname{Length}\left(S^{k}\right)
$$

and it is determined by the marked length spectrum of $M^{n}$.

In the proof of this theorem, we observed that the length of every element of $\pi_{1}$ was achieved within the soul. Combining this with our Theorem [3.6. we see that either there are only finitely many elements in the fundamental group or the universal cover contains a line. This was already shown in the compact setting by Cheeger-Gromoll [7].

Theorem 7.2 [37] If $M^{n}$ is a complete noncompact Alexandrov space with nonnegative curvature, then immediately

$$
\operatorname{Shift}\left(M^{n}\right)=\operatorname{Length}\left(M^{n}\right),
$$

the slipping group and universal slipping groups are trivial.

We can also prove the following theorem relating the holonomy to the covering spectrum:

Theorem 7.3 If a complete noncompact manifold, $M^{m}$, with nonnegative sectional curvature has a connected holonomy group, then the fundamental group lies in the rescaled slipping group and the infinite rescaled covering spectrum is empty. More generally, if $g \in \pi_{1}\left(M^{n}\right)$ has a representative loop whose holonomy lies in the identity component of the holonomy group then $\sup \{L(g, p): p \in$ $\left.M^{m}\right\}<\infty$ and $g$ lies in the rescaled slipping group.

Proof: Perelman proved that for all $t>0$, we have

$$
d_{M}\left(\exp _{q_{1}}\left(t v_{1}\right), \exp _{q_{2}}\left(t v_{2}\right)\right) \geq d_{S}\left(q_{1}, q_{2}\right)
$$

as long as $v_{i} \in T M_{q_{i}}$ are perpendicular to the soul. Furthermore, he proved that if there is a path from $q_{1}$ to $q_{2}$ such that $v_{1}$ is parallel to $v_{2}$ along that path, then there is equality for all $t>0$ [27] [28].

Now suppose $g \in \pi_{1}(M)$ has a representative loop, $C$, which lies in the identity component of the holonomy group of $M^{n}$. We apply the distance decreasing retraction, $P$, to obtain a loop $P \circ C$, in the soul which is freely homotopic to $C$, and thus also has holonomy in the identity component of the holonomy group of $M^{n}$. Recall the identity component of the holonomy is generated by contractible loops. So in this case one can compose $P \circ C$ with a trivial loop $\gamma_{0}$ so that the composition, $\gamma:[0,1] \rightarrow S \subset M$, has a closed parallel normal vector field, $v(s)$, such that $v(0)=v(1)$. Thus, taking $C_{t}(s)=\exp _{\gamma(s)}(t v(s))$ as our representative of $g$ based at $p_{t}=C_{t}(0)$ we have

$$
L\left(g, p_{t}\right) \leq L\left(C_{t}\right)=L(\gamma)
$$


This

$$
L_{r s}^{\infty}(g) \leq \liminf _{t \rightarrow \infty} \frac{L\left(g, p_{t}\right)}{t}=0
$$

Remark 7.4 Note that the infinite Moebius strip has holonomy group, $\mathbb{Z}_{2}$, and the value 1 lies in its infinite rescaled covering spectrum. One might think that any complete noncompact manifold with nonnegative sectional curvature that has a nonempty infinite rescaled covering spectrum has a line in its universal cover. However, in the appendix we will show that Wilking's example has a nontrivial covering spectrum.

\subsection{Positive Ricci curvature and Theorem 1.1}

For many years after Cheeger and Gromoll proved the Soul Theorem 8 for manifolds with nonnegative sectional curvature, it was an open question whether a complete noncompact manifold with positive Ricci curvature must have a finite fundamental group. After all, if the manifold has positive Ricci curvature and nonnegative sectional curvature, then it has a soul with positive Ricci curvature and the fundamental group must be finite by Myers' Theorem [23. Manifolds with positive sectional curvature have trivial fundamental groups.

Then in 1980, Nabonnand 24 found an example of a complete noncompact manifold with positive Ricci curvature whose fundamental group is $\mathbb{Z}$. In fact, in [39], the second author showed that fundamental group could be any torsion free nilpotent group and Wilking adapted these examples to prove the fundamental group could be any almost nilpotent group [40.

Below we will describe Nabonnand's example in more detail and will see below in Proposition 7.7 . that all elements of the fundamental group of his example lie in the rescaled slipping group. We will also study the Berárd-Bergery Examples which demonstrate a variety of covering spectra can be achieved on manifolds with positive Ricci curvature [Proposition 7.11. In the Appendix we will see that Wilking's example has positive Ricci curvature and a nontrivial rescaled covering spectrum.

First we prove Theorem [1.1, stated in the introduction, that for any compact set $K$ in a complete noncompact manifold with positive Ricci curvature, there are only finitely many distinct elements $g$ of $\pi_{1}(M)$ such that $L(g)$ are achieved in the compact set $K$.

Proof: By Theorem [3.6. we know that if there are infinitely many elements whose length is achieved within a compact set, then the universal cover contains a line. However, by the Cheeger-Gromoll Splitting Theorem [8], any manifold with nonnegative Ricci curvature that contains a line splits isometrically, and thus cannot have strictly positive Ricci curvature.

A corollary of Theorem 1.1 is:

Corollary 7.5 Let $M$ be a complete Riemannian manifold with Ric $>0$. If there is a compact set $K \subset M$ such that $L(g, p)$ is nondecreasing outside of $K$ as $p \rightarrow \infty$, then $\pi_{1}(M)$ is finite.

Remark 7.6 Note that the nondecreasing hypothesis in Corollary 7.5 implies that the slipping group is trivial and the covering spectrum has an infimum. One may ask if one still must have a finite fundamental group when the nondecreasing hypothesis is replaced by an assumption that the slipping group is trivial and the covering spectrum has an infimum. However we will produce many examples in Proposition 7.11 which have infinite fundamental groups.

Then Berárd-Bergery [2] showed that, given any compact manifold $M^{m}$ with Ricci $\geq 0, N^{m+3}=$ $M^{m} \times \mathbb{R}^{3}$ has a complete warping metric

$$
g=d r^{2}+h^{2}(r) g_{\mathbb{S}^{2}}+f^{2}(r) g_{M} \text { with } h(0)=0, h^{\prime}(0)=1, \text { and } f(0) \neq 0, f^{\prime}(0)=0 .
$$


with Ric $>0$. Nabonnand's example had the same structure with $M=\mathbb{S}^{1}$.

For complete manifolds with Ric $\geq 0$, unlike manifolds with nonnegative sectional curvature, $L(g, p)$ could decrease as $p$ goes to infinity. In Nabannand's example [24] of $\mathbb{S}^{1} \times \mathbb{R}^{3}$ with Ric $>0$, the length of $\mathbb{S}^{1}$ strictly decreases as it goes to infinity. In fact we can show that:

Proposition 7.7 For any warping metric as in 7.119) (with $M=\mathbb{S}^{1}$ ) on $N=\mathbb{S}^{1} \times \mathbb{R}^{3}$ ), if Ric $>0$, then $f^{\prime}(r)<0$ for $r>0$. So the rescaled covering spectrum is empty and $\pi_{1}(N)$ is contained in the rescaled slipping group.

Proof: To see this we examine the Ricci curvature in the $\mathbb{S}^{1}$ direction, $V$, which, according to Nabonnand's equation (1) is

$$
-\operatorname{Ric}(V, V)=\frac{f^{\prime \prime}(r)}{f(r)}+2 \frac{f^{\prime}(r) h^{\prime}(r)}{f(r) h(r)}<0 \quad \text { for } r>0 .
$$

By smoothness requirements $f^{\prime}(0)=0$ and $f(r)>0$ for all $r \in[0, \infty)$. Suppose $f^{\prime}\left(r_{0}\right)=0$. Then

$$
\frac{f^{\prime \prime}\left(r_{0}\right)}{f\left(r_{0}\right)}=\frac{f^{\prime \prime}\left(r_{0}\right)}{f\left(r_{0}\right)}+2 \frac{f^{\prime}\left(r_{0}\right) h^{\prime}\left(r_{0}\right)}{f\left(r_{0}\right) h\left(r_{0}\right)}<0 .
$$

So $f^{\prime \prime}\left(r_{0}\right)<0$ and all critical points are local maximal. This implies there is at most one critical point and $f^{\prime}(r)<0$ after that critical point. Since $f^{\prime}(0)=0$ we have $f^{\prime}(r)<0$ for all $r>0$. So $f$ is strictly decreasing.

Any $g \in \pi_{1}(N)$ is also a $g \in \pi_{1}(M)$ with such a construction and any point in $N$ can be represented as $(x, r)$ with $x \in M$. So applying Definition 5.1

$$
\begin{aligned}
L_{r s}^{\infty}(g) \leq L_{r s}^{x_{0}}(g) & =\inf _{(x, r) \in N \backslash\left\{\left(x_{0}, 0\right)\right\}} \frac{d_{\tilde{N}}(g(\tilde{x, r}),(\tilde{x}, r))}{d_{N}\left((x, r),\left(x_{0}, 0\right)\right)} \\
& \leq \lim _{r \rightarrow \infty} \frac{f(r) d_{\tilde{M}}(g \tilde{x}, \tilde{x})}{r-d_{N}\left(x, x_{0}\right)}=0 .
\end{aligned}
$$

Remark 7.8 The above theorem also holds for general warped products as in (7.119) when $M^{n}$ has nonnegative Ricci curvature, e.g. $M^{n}=T^{n}$.

Remark 7.9 Note that in general the rescaled slipping group of a manifold with Ricci $>0$ may not be the entire fundamental group even though such manifolds have the loops to infinity property. See Example 8.1 in the appendix.

Remark 7.10 In Berárd-Bergery type examples where $f(r)$ is monotone decreasing in $r$ in (7.119), $\lim _{r \rightarrow \infty} f(r)$ could be zero or any positive number. When $\lim _{r \rightarrow \infty} f(r)=0$, the entire fundamental group is in the slipping group and the universal delta cover is the whole space.

Proposition 7.11 If $M^{n}$ is a compact manifold with nonnegative Ricci curvature, then there is a Berárd-Bergery example, $N^{n+3}$ of a complete noncompact manifold with positive Ricci curvature as in (7.119) such that

$$
\operatorname{Cov} \operatorname{Spec}\left(N^{n+3}\right)=\operatorname{Cov} \operatorname{Spec}\left(M^{n}\right) .
$$

Proof: In the Berárd-Bergery examples, $f(r)$ need only be decreasing and we can find decreasing $f(r)$ such that $\lim _{r \rightarrow \infty} f(r)=1$.

Remark 7.12 If $M^{n}$ has positive Ricci curvature, then $f$ need not be strictly decreasing to make $M \times \mathbb{R}^{3}$ have positive Ricci curvature. One can take $f(r)$ to be constant and $h(r)$ concave down, since the isometric product of $M$ with $\mathbb{R}^{3}$ where $\mathbb{R}^{3}$ has Ric $>0$ would have positive Ricci curvature. Then we get CovSpec $\left(M \times \mathbb{R}^{3}\right)=\operatorname{CovSpec}(M)$ and $\operatorname{CovSpec} \operatorname{Sps}_{r s}^{x_{0}}\left(M \times \mathbb{R}^{3}\right)=0$. 


\subsection{Ricci curvature and the rescaled covering spectrum}

We now prove Theorem 7.13 . In fact we prove the following stronger theorem:

Theorem 7.13 Suppose $M^{n}$ is a complete noncompact manifold, $M^{n}$, and $\pi_{r s}^{\infty}(M, \delta)$ is trivial. If $M^{n}$ has nonnegative Ricci curvature then the fundamental group is finite with less than $2(2+\delta)^{n} / \delta^{n}$ elements.

Proof: Assume on the contrary there are $N$ nontrivial elements $g_{1}, g_{2}, \ldots g_{N-1} \in \pi_{1}(M)$ such that $g_{i} \neq g_{j}^{ \pm 1}$ for all $i \neq j$. Let $g_{0}$ be the identity in $\pi_{1}(M)$. When $M$ has nonnegative Ricci curvature, we have our theorem if we prove $N \leq \frac{(2+\delta)^{n}}{\delta^{n}}$. So we assume on the contrary that

$$
N>\frac{(2+\delta)^{n}}{\delta^{n}} \text {. }
$$

For all $i, j \in\{0,1,2, \ldots(N-1)\}$ with $i \neq j$, we have $g_{i} g_{j}^{-1}$ are nontrivial. So

$$
g_{i} g_{j}^{-1} \notin \pi_{r s}^{\infty}(M, \delta)
$$

and

$$
L_{r s}^{\infty}\left(g_{i} g_{j}^{-1}\right) \geq 2 \delta .
$$

So for any $x_{0} \in M, \epsilon>0$, there exists $R_{i, j, \varepsilon}$ sufficiently large that $\forall R \geq R_{i, j, \varepsilon}$ we have

$$
\inf _{x \in M \backslash B_{x_{0}}(R)} \frac{d_{\tilde{M}}\left(\tilde{x}, g_{i} g_{j}^{-1} \tilde{x}\right)}{d_{M}\left(x, x_{0}\right)}>2 \delta-2 \varepsilon .
$$

Let $R_{\varepsilon}=\max \left\{R_{i, j, \varepsilon}: i, j \in 0,1, \ldots(N-1)\right\}$. Then (17.128) holds for all $R \geq R_{\varepsilon}$.

In particular, for all $x \in M \backslash B_{x_{0}}\left(R_{\varepsilon}\right)$ we have

$$
B_{g_{i} \tilde{x}}\left(\rho_{\varepsilon}(x)\right) \cap B_{g_{j} \tilde{x}}\left(\rho_{\varepsilon}(x)\right)=\emptyset
$$

where

$$
\rho_{\varepsilon}(x)=\rho(x)(\delta-\varepsilon) \text { where } \rho(x)=d\left(x, x_{0}\right) .
$$

Fix a lift of $x_{0}, \tilde{x}_{0}$, choose $\tilde{x}$, a lift of $x$, such that

$$
d_{\tilde{M}}\left(\tilde{x}, \tilde{x}_{0}\right)=d_{M}\left(x, x_{0}\right)=\rho(x) .
$$

We now apply the trick in Milnor's paper [21. First we set

$$
C=\max \left\{d_{\tilde{M}}\left(\tilde{x}_{0}, g_{i} \tilde{x}_{0}\right): i=0,1,2 \ldots N\right\} .
$$

Then by the triangle inequality,

$$
B_{g_{i} \tilde{x}}\left(\rho_{\varepsilon}(x)\right) \subset B_{\tilde{x}_{0}}\left(C+\rho(x)+\rho_{\varepsilon}(x)\right) \subset B_{\tilde{x}}\left(C+2 \rho(x)+\rho_{\varepsilon}(x)\right) .
$$

Since

$$
\operatorname{vol}\left(B_{g_{i} \tilde{x}}\left(\rho_{\varepsilon}(x)\right)\right)=\operatorname{vol}\left(B_{\tilde{x}}\left(\rho_{\varepsilon}(x)\right)\right.
$$

and the balls are disjoint, we have

$$
N \operatorname{vol}\left(B_{\tilde{x}}\left(\rho_{\varepsilon}(x)\right) \leq \operatorname{vol}\left(B_{\tilde{x}}\left(C+2 \rho(x)+\rho_{\varepsilon}(x)\right) .\right.\right.
$$

By the Bishop-Gromov Volume Comparison Theorem, we have

$$
N \leq \frac{\left(C+2 \rho(x)+\rho_{\varepsilon}(x)\right)^{n}}{\left(\rho_{\varepsilon}(x)\right)^{n}} .
$$


Substituting $\rho_{\varepsilon}(x)=\rho(x)(\delta-\varepsilon)$ we have

$$
N \leq \frac{(C+\rho(x)(2+\delta-\varepsilon))^{n}}{(\rho(x)(\delta-\varepsilon))^{n}}
$$

for all $x \in M \backslash B_{x_{0}}\left(R_{\varepsilon}\right)$. So now we take $x \rightarrow \infty, \rho(x) \rightarrow \infty$ and we have

$$
N \leq \frac{(2+\delta-\varepsilon)^{n}}{(\delta-\varepsilon)^{n}}
$$

Lastly we take $\varepsilon \rightarrow 0$ and we have our contradiction.

Remark 7.14 A version of Theorem 1.2 holds for any metric measure space whose universal cover satisfies a doubling condition for arbitrarily large radii including the pointed Gromov-Hausdorff limits of manifolds with nonnegative Ricci curvature. In that case we can prove the group of deck transforms of the universal covering space is finite. See [34] for similar discussions of extensions of theorems regarding the universal covers of manifolds with nonnegative Ricci curvature to their limit spaces.

Remark 7.15 It should be noted that Anderson extended Milnor's techniques by intersecting balls with fundamental domains and taking their radii to infinity in [1]. He proved that the fundamental group in a manifold with nonnegative Ricci curvature and $\liminf _{R \rightarrow \infty} \operatorname{vol}\left(B_{x_{0}}(R)\right) / R^{n}=\alpha>0$ has a fundamental group which is finite with $\leq \omega_{n} / \alpha$ elements. Li also proved this using the heat kernal in [19]. One might ask if manifolds satisfying the conditions in their theorem have a trivial rescaled covering group for some value $\delta$. Or perhaps, one could show that at least the rescaled slipping group is trivial.

Remark 7.16 The first author proved in [31] that the fundamental group of a manifold, $M^{n}$, with nonnegative Ricci curvature and linear volume growth

$$
\limsup _{R \rightarrow \infty} \frac{\operatorname{vol}\left(B_{x_{0}}(R)\right)}{R}<\infty
$$

has a finitely generated fundamental group. One might ask whether under these conditions one can prove there is a $\delta>0$ such that $\pi_{r s}^{\infty}(M, \delta)$ is trivial and thus improve this theorem to imply that the fundamental group is finite. However the cylinder and the infinite Moebius strip both have linear volume growth (and 0 curvature), and infinite fundamental group with a nontrivial rescaled slipping group and so $\pi_{r s}^{\infty}(\delta)$ is nontrivial for all $\delta>0$.

Remark 7.17 The first author proved in [31] that the fundamental group of a manifold, $M^{n}$, with nonnegative Ricci curvature with small linear diameter growth

$$
\limsup _{R \rightarrow \infty} \frac{\operatorname{diam}\left(B_{x_{0}}(R)\right)}{R} \leq S_{n}
$$

has a finitely generated fundamental group. The constant provided in [31] was improved by [43]. One might ask whether under these conditions one can prove there is a $\delta>0$ such that $\pi_{r s}^{\infty}(M, \delta)$ is trivial and thus improve this theorem to imply that the fundamental group is finite. However, this is not possible: Nabonnand's example has both an infinite fundamental group and sublinear diameter growth as well as a nontrivial rescaled slipping group. 


\section{Appendix}

We would like to thank Burkhard Wilking for suggesting this example when asked for an example of a manifold with nonnegative sectional curvature that had $L(g, p)$ diverging to infinity as $p \rightarrow \infty$, but the universal cover has no line.

Example 8.1 Let $M^{6}=\left(\mathbb{S}^{3} \times \mathbb{R}^{4}\right) / \operatorname{Pin}(2)$. This has nonnegative sectional curvature because it is a quotient of a manifold with nonnegative sectional curvature. It is $\mathbb{R}^{4}$ bundle with soul $\mathbb{S}^{3} / \operatorname{Pin}(2)=$ $\mathbb{R} P^{2}$.

In Proposition 8.2 we will prove this example has positive Ricci curvature. In Proposition 8.3 . we will prove the infinite rescaled covering spectrum is nontrivial. These are the only facts needed to apply this example earlier in the paper.

Before studying the properties of this example, we explain the construction in more detail. Here $\mathbb{S}^{3} \times \mathbb{R}^{4}$ is the standard isometric product with nonnegative sectional curvature. One takes

$$
\mathbb{S}^{3} \times \mathbb{R}^{4}=\left\{\left(z_{1}, z_{2}, z_{3}, z_{4}\right) \in \mathbb{C}^{4}:\left|z_{1}\right|^{2}+\left|z_{2}\right|^{2}=1\right\} .
$$

We can take an $\mathbb{S}^{1}$ action on this space:

$$
e^{i \theta}\left(z_{1}, z_{2}, z_{3}, z_{4}\right)=\left(e^{i \theta} z_{1}, e^{i \theta} z_{2}, e^{i \theta} z_{3}, e^{i \theta} z_{4}\right) .
$$

Then $\tilde{M}^{6}=\left(\mathbb{S}^{3} \times \mathbb{R}^{4}\right) / \mathbb{S}^{1}$ has nonnegative sectional curvature by O'Neill's Submersion Formula [26]. The elements of $\tilde{M}^{6}$ are equivalence classes

$$
\left[\left(z_{1}, z_{2}, z_{3}, z_{4}\right)\right]=\left[\left(e^{i \theta} z_{1}, e^{i \theta} z_{2}, e^{i \theta} z_{3}, e^{i \theta} z_{4}\right)\right] \in \tilde{M}^{6} .
$$

Recall that the Hopf sphere has $\mathbb{S}^{2}=\mathbb{S}^{3} / \mathbb{S}^{1}$, where we also view

$$
\mathbb{S}^{3}=\left\{\left(z_{1}, z_{2}\right) \in \mathbb{C}^{2}:\left|z_{1}\right|^{2}+\left|z_{2}\right|^{2}=1\right\}
$$

with the same kind of circle action

$$
e^{i \theta}\left(z_{1}, z_{2}\right)=\left(e^{i \theta} z_{1}, e^{i \theta} z_{2}\right) .
$$

The equivalence classes

$$
\left[\left(x_{1}+i x_{3}, x_{2}+i x_{4}\right)\right]=\left[\left(z_{1}, z_{2}\right)\right]=\left[\left(e^{i \theta} z_{1}, e^{i \theta} z_{2}\right)\right] \in \mathbb{S}^{3} / \mathbb{S}^{1}
$$

are identified with points

$$
\left(2\left(x_{1} x_{2}+x_{3} x_{4}\right), 2\left(x_{1} x_{4}-x_{2} x_{3}\right), x_{1}^{2}+x_{3}^{2}-x_{2}^{2}-x_{4}^{2}\right) \in\left\{(a, b, c): a^{2}+b^{2}+c^{2}=1\right\}=\mathbb{S}^{2} \subset \mathbb{R}^{3} .
$$

We can see that $\tilde{M}^{6}$ is an $\mathbb{R}^{4}$ bundle over $\mathbb{S}^{2}$ with

$$
\left.\pi\left(\left[\left(e^{i \theta} z_{1}, e^{i \theta} z_{2}\right), e^{i \theta} z_{3}, e^{i \theta} z_{4}\right)\right]\right)=\left[\left(e^{i \theta} z_{1}, e^{i \theta} z_{2}\right)\right]
$$

So it is simply connected.

We define $M^{6}=\tilde{M}^{6} / \mathbb{Z}_{2}$ taking the antipodal map on the $\mathbb{S}^{2}$ base and the fibers. First note that the antipodal map, $g_{0}$, on $\mathbb{S}^{2}$ :

$$
\begin{aligned}
g_{0}\left[\left(e^{i \theta} z_{1}, e^{i \theta} z_{2}\right)\right] & =g_{0}\left[\left(x_{1}+i x_{3}, x_{2}+i x_{4}\right)\right] \\
& =g_{0}\left(2\left(x_{1} x_{2}+x_{3} x_{4}\right), 2\left(x_{1} x_{4}-x_{2} x_{3}\right), x_{1}^{2}+x_{3}^{2}-x_{2}^{2}-x_{4}^{2}\right) \\
& =\left(-2\left(x_{1} x_{2}+x_{3} x_{4}\right),-2\left(x_{1} x_{4}-x_{2} x_{3}\right),-x_{1}^{2}-x_{3}^{2}+x_{2}^{2}+x_{4}^{2}\right) \\
& =\left[\left(x_{2}-i x_{4},-x_{1}+x_{3} i\right)\right]=\left[\left(\bar{z}_{2},-\bar{z}_{1}\right)\right] \\
& =\left[\left(e^{i \theta} \bar{z}_{2}, e^{i(\pi+\theta)} \bar{z}_{1}\right)\right]
\end{aligned}
$$


We define the $\mathbb{Z}_{2}$ action $g$ on $\tilde{M}^{6}$ such that $\pi \circ g=g \circ g_{0}$ as follows:

$$
g\left[\left(e^{i \theta} z_{1}, e^{i \theta} z_{2}, e^{i \theta} z_{3}, e^{i \theta} z_{4}\right)\right]=\left[\left(e^{i \theta} \bar{z}_{2}, e^{i(\pi+\theta)} \bar{z}_{1}, e^{i \theta} \bar{z}_{4}, e^{i(\pi+\theta)} \bar{z}_{3}\right)\right] .
$$

Since the soul of $\tilde{M}^{6}=\mathbb{S}^{3} \times \mathbb{R}^{4} / \mathbb{S}^{1}$, is $\mathbb{S}^{3} / \mathbb{S}^{1}=\mathbb{S}^{2}$, we know the soul of $M^{6}=\mathbb{S}^{3} \times \mathbb{R}^{4} / \operatorname{Pin}(2)=$ $\tilde{M}^{6} / \mathbb{Z}_{2}$ is $\mathbb{R} P^{2}=\mathbb{S}^{2} / \mathbb{Z}_{2}$. Wilking made an argument using this soul and the tangent cone at infinity to show the universal covering space does not contain a line. However, we see here that it does not contain a line because in fact it has positive Ricci curvature:

Proposition 8.2 The Wilking Example, $M^{6}$, has positive Ricci curvature.

Proof: We will examine $\tilde{M}^{6}$. The soul directions have positive Ricci curvature because the soul has positive sectional curvature and the total space has nonnegative sectional curvature. So we only need to check the fibre directions. The fibres are totally geodesic with quotient metric $\mathbb{R}^{4} \times \mathbb{S}^{1} / \mathbb{S}^{1}$. We can write the metric of $\mathbb{R}^{4} \times \mathbb{S}^{1}$ as

$$
d r^{2}+r^{2}\left(\left(\sigma^{1}\right)^{2}+\left(\sigma^{2}\right)^{2}+\left(\sigma^{3}\right)^{2}+d \theta^{2},\right.
$$

where $\left(\sigma^{1}\right)^{2}+\left(\sigma^{2}\right)^{2}+\left(\sigma^{3}\right)^{2}$ is the standard left invariant metric on $\mathbb{S}^{3}$, with dual orthonormal vector fields $X_{1}, X_{2}, X_{3}$ satisfy the bracket relation $\left[X_{i}, X_{i+1}\right]=2 X_{i+2}$ (indices are mod 3$)$. Let $X_{1}$ be tangent to the Hopf fibre direction. Then the orbit direction of $\mathbb{S}^{1}$ acts on $\mathbb{R}^{4} \times \mathbb{S}^{1}$ is $X_{1}+\frac{\partial}{\partial \theta}$. Therefore the orthonormal basis of $\mathbb{R}^{4} \times \mathbb{S}^{1} / \mathbb{S}^{1}$ is $\frac{\partial}{\partial r}, \bar{X}_{1}=\frac{1}{\sqrt{1+r^{2}}}\left(\frac{1}{r} X_{1}-r \frac{\partial}{\partial \theta}\right), \bar{X}_{2}=\frac{1}{r} X_{2}, \bar{X}_{3}=\frac{1}{r} X_{3}$. Now we can use O'Neill's formula to compute the sectional curvatures. One can find $K\left(\frac{\partial}{\partial r}, \bar{X}_{1}\right)=$ $\left\|\left[\frac{\partial}{\partial r}, \bar{X}_{1}\right]^{v}\right\|^{2}=\frac{4}{\left(1+r^{2}\right)^{2}}$, and $K\left(\bar{X}_{2}, \bar{X}_{3}\right)=\left\|\left[\bar{X}_{2}, \bar{X}_{3}\right]^{v}\right\|^{2}=\frac{4}{1+r^{2}}$. Therefore the Ricci curvature of all directions are positive.

Intuitively, one can see that $L(g, p) \rightarrow \infty$ and $p \rightarrow \infty$ in $M^{6}$. However, we will now prove it grows linearly to obtain the following proposition:

Proposition 8.3 The Wilking Example, $M^{6}$, has nontrivial infinite rescaled covering spectrum.

Proof: The fundamental group of $M^{6}$ is $\mathbb{Z}_{2}$. Let $g$ be the nontrivial element, described by its action on $\tilde{M}$ in (8.14). We need only show:

$$
L_{r s}^{\infty}(g)=\lim _{R \rightarrow \infty} \inf _{x \in M^{6} \backslash B_{x_{0}}(R)} \frac{d_{\tilde{M}^{6}}(g \tilde{x}, \tilde{x})}{d_{M^{6}}\left(x, x_{0}\right)}>0 .
$$

First we compute:

$$
\begin{aligned}
d_{\tilde{M}^{6}}(g \tilde{x}, \tilde{x}) & =d_{\tilde{M}^{6}}\left(g\left[\left(z_{1}, z_{2}, z_{3}, z_{4}\right)\right],\left[\left(z_{1}, z_{2}, z_{3}, z_{4}\right)\right]\right) \\
& =d_{\tilde{M}^{6}}\left(\left[\left(e^{i \theta} \bar{z}_{2}, e^{i(\pi+\theta)} \bar{z}_{1}, e^{i \theta} \bar{z}_{4}, e^{i(\pi+\theta)} \bar{z}_{3}\right)\right],\left[\left(e^{i \theta} z_{1}, e^{i \theta} z_{2}, e^{i \theta} z_{3}, e^{i \theta} z_{4}\right)\right]\right) \\
& =\inf _{\theta, \phi} d_{\mathbb{S}^{3} \times \mathbb{R}^{4}}\left(\left(e^{i \theta} \bar{z}_{2}, e^{i(\pi+\theta)} \bar{z}_{1}, e^{i \theta} \bar{z}_{4}, e^{i(\pi+\theta)} \bar{z}_{3}\right),\left(e^{i \phi} z_{1}, e^{i \phi} z_{2}, e^{i \phi} z_{3}, e^{i \phi} z_{4}\right)\right) \\
& =\inf _{\theta, \phi} d_{\mathbb{S}^{3} \times \mathbb{R}^{4}}\left(\left(e^{i(\theta-\phi)} \bar{z}_{2}, e^{i(\pi+\theta-\phi)} \bar{z}_{1}, e^{i(\theta-\phi)} \bar{z}_{4}, e^{i(\pi+\theta-\phi)} \bar{z}_{3}\right),\left(z_{1}, z_{2}, z_{3}, z_{4}\right)\right) \\
& =\inf _{\theta^{\prime}} d_{\mathbb{S}^{3} \times \mathbb{R}^{4}}\left(\left(e^{i \theta^{\prime}} \bar{z}_{2}, e^{i\left(\pi+\theta^{\prime}\right)} \bar{z}_{1}, e^{i \theta^{\prime}} \bar{z}_{4}, e^{i\left(\pi+\theta^{\prime}\right)} \bar{z}_{3}\right),\left(z_{1}, z_{2}, z_{3}, z_{4}\right)\right) \\
& =\inf _{\theta^{\prime}} \sqrt{\left(d_{\mathbb{S}^{3}}\left(\left(e^{i \theta^{\prime}} \bar{z}_{2}, e^{i\left(\pi+\theta^{\prime}\right)} \bar{z}_{1}\right),\left(z_{1}, z_{2}\right)\right)\right)^{2}+\left(d_{\mathbb{R}^{4}}\left(\left(e^{i \theta^{\prime}} \bar{z}_{4}, e^{i\left(\pi+\theta^{\prime}\right)} \bar{z}_{3}\right),\left(z_{3}, z_{4}\right)\right)\right)^{2}} \\
& \geq \inf _{\theta^{\prime}} d_{\mathbb{R}^{4}}\left(\left(e^{i \theta^{\prime}} \bar{z}_{4}, e^{i\left(\pi+\theta^{\prime}\right)} \bar{z}_{3}\right),\left(z_{3}, z_{4}\right)\right) \\
& =\inf _{\theta^{\prime}} \sqrt{\left|e^{i \theta^{\prime}} \bar{z}_{4}-z_{3}\right|^{2}+\left|e^{i\left(\pi+\theta^{\prime}\right)} \bar{z}_{3}-z_{4}\right|^{2}}
\end{aligned}
$$




$$
\begin{aligned}
& \geq \inf _{\theta^{\prime}} \frac{\sqrt{2}}{2}\left(\left|e^{i \theta^{\prime}} \bar{z}_{4}-z_{3}\right|+\left|e^{i\left(\pi+\theta^{\prime}\right)} \bar{z}_{3}-z_{4}\right|\right) \\
& =\inf _{\theta^{\prime}} \frac{\sqrt{2}}{2}\left(\left|e^{-i \theta^{\prime}} z_{4}-\bar{z}_{3}\right|+\left|e^{i\left(\pi+\theta^{\prime}\right)} \bar{z}_{3}-z_{4}\right|\right) \\
& =\inf _{\theta^{\prime}} \frac{\sqrt{2}}{4}\left(\left|z_{4}-e^{i \theta^{\prime}} \bar{z}_{3}\right|+\left|e^{i\left(\pi+\theta^{\prime}\right)} \bar{z}_{3}-z_{4}\right|+\left|e^{-i \theta^{\prime}} z_{4}-\bar{z}_{3}\right|+\left|\bar{z}_{3}-e^{-i\left(\pi+\theta^{\prime}\right)} z_{4}\right|\right) \\
& \geq \inf _{\theta^{\prime}} \frac{\sqrt{2}}{4}\left(\left|e^{i \theta^{\prime}} \bar{z}_{3}-e^{i\left(\pi+\theta^{\prime}\right)} \bar{z}_{3}\right|+\left|e^{-i \theta^{\prime}} z_{4}-e^{-i\left(\pi+\theta^{\prime}\right)} z_{4}\right|\right) \\
& =\inf _{\theta^{\prime}} \frac{\sqrt{2}}{4}\left(\left|\bar{z}_{3}-e^{i \pi} \bar{z}_{3}\right|+\left|z_{4}-e^{-i \pi} z_{4}\right|\right) \\
& =\frac{\sqrt{2}}{4}\left(\left|1-e^{i \pi}\right|\left|\bar{z}_{3}\right|+\left|1-e^{-i \pi}\right|\left|z_{4}\right|\right) \\
& =\frac{\sqrt{2}}{4}\left(2\left|\bar{z}_{3}\right|+2\left|z_{4}\right|\right) \\
& \geq \frac{\sqrt{2}}{2} \sqrt{\left|z_{3}\right|^{2}+\left|z_{4}\right|^{2}} .
\end{aligned}
$$

On the other hand

$$
d_{M^{6}}\left(x, x_{0}\right)=\min \left\{d_{\tilde{M}^{6}}\left(\tilde{x}, \tilde{x}_{0}\right), d_{\tilde{M}^{6}}\left(\tilde{x}, g \tilde{x}_{0}\right)\right\}
$$

So by the triangle inequality

$$
\left|d_{M^{6}}\left(x, x_{0}\right)-d_{\tilde{M}^{6}}\left(\tilde{x}, \tilde{x}_{0}\right)\right| \leq d_{\tilde{M}^{6}}\left(\tilde{x}_{0}, g \tilde{x}_{0}\right)
$$

Choosing $x_{0}$ in the soul, $\tilde{x}_{0}=\left[\left(w_{1}, w_{2}, 0,0\right)\right]$, so

$$
\begin{aligned}
d_{\tilde{M}^{6}}\left(\tilde{x}, \tilde{x}_{0}\right) & =\inf _{\theta, \phi} d_{\mathbb{S}^{3} \times \mathbb{R}^{4}}\left(\left(e^{i \theta} w_{1}, e^{i \theta} w_{2}, 0,0\right),\left(e^{i \phi} z_{1}, e^{i \phi} z_{2}, e^{i \phi} z_{3}, e^{i \phi} z_{4}\right)\right) \\
& \leq d_{\mathbb{S}^{3} \times \mathbb{R}^{4}}\left(\left(w_{1}, w_{2}, 0,0\right),\left(z_{1}, z_{2}, z_{3}, z_{4}\right)\right) \\
& \leq \sqrt{\pi^{2}+\left|z_{3}\right|^{2}+\left|z_{4}\right|^{2}} .
\end{aligned}
$$

If $d_{M^{6}}\left(x, x_{0}\right) \rightarrow \infty$, then $d_{\tilde{M}^{6}}\left(\tilde{x}, \tilde{x}_{0}\right) \rightarrow \infty$ and $\left|z_{3}\right|^{2}+\left|z_{4}\right|^{2} \rightarrow \infty$. Thus

$$
\begin{aligned}
L_{r s}^{\infty}(g) & =\lim _{R \rightarrow \infty} \inf _{x \in M^{6} \backslash B_{x_{0}}(R)} \frac{d_{\tilde{M}^{6}}(g \tilde{x}, \tilde{x})}{d_{X}\left(x, x_{0}\right)} \\
& \geq \liminf _{\left|z_{3}\right|^{2}+\left|z_{4}\right|^{2} \rightarrow \infty} \frac{\frac{\sqrt{2}}{2} \sqrt{\left|z_{3}\right|^{2}+\left|z_{4}\right|^{2}}}{\sqrt{\pi^{2}+\left|z_{3}\right|^{2}+\left|z_{4}\right|^{2}}+d_{\tilde{M}^{6}}\left(\tilde{x}_{0}, g \tilde{x}_{0}\right)}=\sqrt{2} / 2 .
\end{aligned}
$$

Thus the rescaled covering spectrum is nontrivial.

\section{References}

[1] Michael T. Anderson. On the topology of complete manifolds of nonnegative Ricci curvature. Topology, 29(1):41-55, 1990.

[2] L. Bérard-Bergery, Quelques exemples de varétés riemanniennes complètes non compactes à courbure de Ricci positive. (French. English summary) [Some examples of noncompact complete Riemannian manifolds with positive Ricci curvature] C. R. Acad. Sci. Paris Sér. I Math. 302 (1986), no. 4, 159-161. 
[3] R. L. Bishop and B. O'Neill, Manifolds of negative curvature, Trans. of. Amer. Math. Soc. 145(1969) 1-49.

[4] D. Burago, Y. Burago, S. Ivanov, A Course in Metric Geometry. Graduate Studies in Mathematics Vol. 33, AMS, 2001.

[5] J. Cheeger, T. Colding, On the structure of spaces with Ricci curvature bounded below I, J. Diff. Geom. 46 (1997) 406-480.

[6] J. Cheeger, T. Colding, On the structure of spaces with Ricci curvature bounded below III, J. Differential Geom. 54 (2000), no. 1, 37-74.

[7] Jeff Cheeger and Detlef Gromoll. The splitting theorem for manifolds of nonnegative Ricci curvature. J. Differential Geometry 6 (1971/72), 119-128.

[8] J. Cheeger, D. Gromoll, On the structure of complete manifolds of nonnegative curvature, Ann. of Math. (2) 96 (1972), 413-443.

[9] Colding, Tobias Holck; Naber, Aaron Sharp Holder continuity of tangent cones for spaces with a lower Ricci curvature bound and applications. Ann. of Math. (2) 176 (2012), no. 2, $1173-1229$.

[10] Y. Colin de Verdiere, Spectre du laplacien et longueurs des géodésiques périodiques. I, II. (French) Compositio Math. 27 (1973), 83-106; ibid. 27 (1973), 159-184.

[11] Jim Conant, Victoria Curnutte, Corey Jones, Conrad Plaut, Kristen Pueschel, Maria Walpole, Jay Wilkins Discrete Homotopy Theory and Critical Values of Metric Spaces, to appear in Fundamenta Mathenaticae, arXiv:1205.2925, May 2012.

[12] de Smit, Bart; Gornet, Ruth; Sutton, Craig J. Sunada's method and the covering spectrum. J. Differential Geom. 86 (2010), no. 3, 501-537.

[13] de Smit, Bart; Gornet, Ruth; Sutton, Craig J. Isospectral surfaces with distinct covering spectra via Cayley graphs. Geom. Dedicata 158 (2012), 343-352.

[14] Yu Ding, Heat kernels and Green's functions on limit spaces. Comm. Anal. Geom. 10 (2002), no. 3, 475-514.

[15] Duistermaat, J. J.; Guillemin, V. W. The spectrum of positive elliptic operators and periodic bicharacteristics. Invent. Math. 29 (1975), no. 1, 39-79

[16] J. Ennis, G. Wei, Describing the universal cover of a compact limit. Differential Geom. Appl. 24 (2006), no. 5, 554-562.

[17] M. Gromov, Metric structures for Riemannian and non-Riemannian spaces, PM 152, Birkhauser, 1999.

[18] S. Honda, Bishop-Gromov type inequality on Ricci limit spaces. J. Math. Soc. Japan 63 (2011), no. 2, 419-442.

[19] Peter Li. Large time behavior of the heat equation on complete manifolds with nonnegative Ricci curvature. Ann. of Math. (2), 124(1):1-21, 1986.

[20] X. Menguy, Examples with bounded diameter growth and infinite topological type. Duke Math. J. 102 (2000), no. 3, 403-412.

[21] J. Milnor, A note on curvature and fundamental group, J. Diff. Geom. 2 (1968) 1-7. 
[22] M. Munn, Volume growth and the topology of pointed Gromov-Hausdorff limits. Differential Geom. Appl. 28 (2010), no. 5, 532-542.

[23] S.B. Myers, Riemannian manifolds with positive mean curvature. Duke Math. J. 8, (1941). 401-404.

[24] P. Nabonnand, Sur les varétés riemanniennes complètes à courbure de Ricci positive. (French. English summary) C. R. Acad. Sci. Paris Sér. A-B 291 (1980), no. 10, A591 $-\mathrm{A} 593$.

[25] Shin-ichi Ohta, On the measure contraction property of metric measure spaces. Comment. Math. Helv. 82 (2007), no. 4, 805-828.

[26] B. O'Neill, The fundamental equations of a submersion, Michigan Math. J. 13 (1966), 459-469.

[27] G. Perelman, Proof of the soul conjecture of Cheeger and Gromoll, J. Differential Geom. 40 (1994), no. 1, 209-212.

[28] G. Perelman, Alexandrov's spaces with curvatures bounded from below II, perpetual preprint.

[29] C. Plaut and J. Wilkins, Discrete homotopies and the fundamental group, Advances in Mathematics 232 (2013), 271-294.

[30] V. A. Sharafutdinov, The Pogorelov-Klingenberg theorem for manifolds homeomorphic to $\mathbb{R}^{n}$, Sib. Math. Zh. 18 (1977) 915-925.

[31] Christina Sormani. Nonnegative Ricci curvature, small linear diameter growth and finite generation of fundamental groups. J. Differential Geom., 54(3):547-559, 2000.

[32] C. Sormani, On Loops Representing Elements of the Fundamental Group of a Complete Manifold with Nonnegative Ricci Curvature, Indiana Journal of Mathematics, 50 (2001) no. $4,1867-1883$.

[33] C. Sormani, Convergence and the length spectrum. Advances in Mathematics 213 (2007), no. $1,405-439$.

[34] C. Sormani and G. Wei, Hausdorff Convergence and Universal Covers, Transactions of the American Mathematical Society 353 (2001) 3585-3602.

[35] C. Sormani and G. Wei, Universal Covers for Hausdorff Limits of Noncompact Spaces, Transactions of the American Mathematical Society 356 (2004) no. 3, 1233-1270.

[36] C. Sormani and G. Wei, The Covering Spectrum of a Compact Length Space, Journal of Differential Geometry, Vol 67 (2004) 35-77.

[37] C. Sormani and G. Wei, The Cut-off Covering Spectrum, Tran. AMS. 362, no. 5 (2010), 2339-2391.

[38] E. Spanier, Algebraic Topology, McGraw-Hill, Inc., 1966.

[39] Guofang Wei. Examples of complete manifolds of positive Ricci curvature with nilpotent isometry groups. Bull. Amer. Math. Soci., 19(1):311-313, 1988.

[40] Burkhard Wilking. On fundamental groups of manifolds of nonnegative curvature. Differential Geom. Appl., 13(2):129-165, 2000. 
[41] J. Wilkins, Discrete Geometric Homotopy Theory and Critical Values of Metric Spaces, Doctoral Dissertation, University of Tennesee Knoxville, 2011.

[42] J Wilkins, The Revised and Uniform Fundamental Groups and Universal Covers of Geodesic Spaces. Topology and its Applications 160 (2013), no. 6, 812-835.

[43] S. Xu, Z. Wang, F. Yang, On the fundamental group of open manifolds with nonnegative Ricci curvature. Chinese Ann. Math. Ser. B 24 (2003), no. 4, 469-474.

Christina Sormani

Department of Mathematics,

CUNY Graduate Center and Lehman College

sormanic@member.ams.org

Guofang Wei,

Department of Mathematics,

University of California Santa Barbara

wei@math.ucsb.edu 